\title{
Iterative algorithms for solutions of Hammerstein equations in real Banach spaces
}

\section{Charles E. Chidume ${ }^{1 *}$, Abubakar Adamu' and Lois C. Okereke ${ }^{1}$}

"Correspondence:

cchidume@aust.edu.ng

${ }^{1}$ African University of Science and

Technology, Abuja, Nigeria

\begin{abstract}
Let $B$ be a uniformly convex and uniformly smooth real Banach space with dual space $B^{*}$. Let $F: B \rightarrow B^{*}, K: B^{*} \rightarrow B$ be maximal monotone mappings. An iterative algorithm is constructed and the sequence of the algorithm is proved to converge strongly to a solution of the Hammerstein equation $u+K F u=0$. This theorem is a significant improvement of some important recent results which were proved in real Hilbert spaces under the assumption that $F$ and $K$ are maximal monotone continuous and bounded. The continuity and boundedness restrictions on $K$ and $F$ have been dispensed with, using our new method, even in the more general setting considered in our theorems. Finally, numerical experiments are presented to illustrate the convergence of the sequence of our algorithm.
\end{abstract}

MSC: 47H09; 47H05; 47J25; 47J05

Keywords: Fixed point; Maximal monotone; Uniformly smooth; Uniformly convex; Hammerstein; Quasi-bounded

\section{Introduction}

Let $B$ be a real Banach space with a strictly convex dual space $B^{*}$. Consider the Hammerstein equation

$$
(I+K F) u=0,
$$

where $F: B \rightarrow B^{*}$ is a nonlinear mapping and $K: B^{*} \rightarrow B$ is a linear map such that $R(F) \subset$ $D(K)$. If $\Omega$ denotes a domain of $\sigma$-finite measure $d y$ in $\mathbb{R}^{N}$, and $\kappa: \Omega \times \Omega \rightarrow \mathbb{R}$ and $f$ : $\Omega \times \mathbb{R} \rightarrow \mathbb{R}$ are measurable real-valued functions on $\Omega$, one can define a linear integral operator $K$ by $K v:=\int_{\Omega} \kappa(\cdot, y) v(y) d y$ and an operator $F$ by the Nemitskyi or superposition operator given by $F u:=f(\cdot, u(\cdot))$ to obtain equation (1.1).

Numerous problems in differential equation theory can, as a rule, be modeled by a Hammerstein equation (see, e.g., Pascali and Sburlan [40]). For example, the amplitude of oscillation $v(t)$ is a solution of the problem

$$
\left\{\begin{array}{l}
\frac{d^{2} v}{d t^{2}}+a^{2} \sin v(t)=z(t), \quad t \in[0,1], \\
v(0)=v(1)=0
\end{array}\right.
$$

(c) The Author(s) 2020. This article is licensed under a Creative Commons Attribution 4.0 International License, which permits use, sharing, adaptation, distribution and reproduction in any medium or format, as long as you give appropriate credit to the original author(s) and the source, provide a link to the Creative Commons licence, and indicate if changes were made. The images or other third party material in this article are included in the article's Creative Commons licence, unless indicated otherwise in a credit line to the material. If material is not included in the article's Creative Commons licence and your intended use is not permitted by statutory regulation or exceeds the permitted use, you will need to obtain permission directly from the copyright holder. To view a copy of this licence, visit http://creativecommons.org/licenses/by/4.0/. 
where the driving force $z(t)$ is periodical and odd. The constant $a \neq 0$ depends on the length of the pendulum and on gravity. Since the Green's function of the problem

$$
v^{\prime \prime}(t)=0, \quad v(0)=v(1)=0
$$

is the triangular function

$$
k(t, x)= \begin{cases}t(1-x), & 0 \leq t \leq x \\ x(1-t), & x \leq t \leq 1\end{cases}
$$

problem (1.2) is equivalent to the nonlinear integral equation

$$
v(t)=-\int_{0}^{1} k(t, x)\left[z(x)-a^{2} \sin v(x)\right] d x .
$$

If $\int_{0}^{1} k(t, x) z(x) d x=g(t)$ and $v(t)+g(t)=u(t)$, then (1.4) can be written as the Hammerstein integral equation

$$
u(t)+\int_{0}^{1} k(t, x) f(x, u(x)) d x=0
$$

where $f(x, u(x))=a^{2} \sin [u(x)-g(x)]$.

The Hammerstein equation also plays a crucial role in the theory of optimal control systems, in automation and in network theory. For example, in studying automatic control, Narendra and Gallman [36], using a Hammerstein model, proposed an iterative method for the identification of nonlinear systems, for samples of inputs and outputs in the presence of noise. For more on problems in optimal control, automation and network systems that can be modeled as Hammerstein equations, see, e.g., Dolezal [30].

Several existence and uniqueness theorems have been proved for equations of Hammerstein type (see, e.g., Brezis and Browder [3, 4], Browder and Gupta [6], Chepanovich [7], De Figueiredo and Gupta [28]).

A map $A: D(A) \subset B \rightarrow B^{*}$ is called monotone if for all $x, y \in B,\langle A x-A y, x-y\rangle \geq 0$. The map $A$ is called maximal monotone if $A$ is monotone, and in addition, $R(J+\lambda A)$ is $B^{*}$, for all $\lambda>0$. Monotone mappings were studied in Hilbert spaces by Zarantonello [46], Minty [35], and a host of other authors. For more recent studies of monotone mappings and Hammerstein equations, the reader may see, for example, Berinde [2], Chidume [8-10], Chidume and Chidume [14], Goebel and Reich [31], Zeidler [47].

In general, equations of Hammerstein type are nonlinear and thus, there are no closedform solutions of such equations. Consequently, methods for approximating such equations are of interest. Several attempts have been made to approximate solutions of equations of Hammerstein type. An early method introduced by Brezis and Browder [5] was in the case where one of the operators is angle bounded (see, e.g., Pascali and Sburlan [40]). They proved strong convergence of a suitably defined Galerkin approximation to a solution of (1.1) as the following theorem.

Theorem 1.1 Let $H$ be a separable Hilbert space and $C$ be a closed subspace of H. Let $K$ : $H \rightarrow C$ be a bounded continuous monotone operator and $F: C \rightarrow H$ be an angle-bounded 
and weakly compact mapping. For a given $f \in C$, consider the Hammerstein equation

$$
(I+K F) u=f
$$

and its nth Galerkin approximation given by

$$
\left(I+K_{n} F_{n}\right) u^{n}=P_{n}^{*} f
$$

with $K_{n}=P_{n}^{*} K P_{n}: H \rightarrow C$ and $F_{n}=P_{n} F P_{n}^{*}: C_{n} \rightarrow H$, where the symbols have their usual meanings (see [40]). Then, for each $n \in N$, the Galerkin approximation (1.6) admits a unique solution $u^{n} \in C_{n}$ and $\left\{u^{n}\right\}$ converges strongly in $H$ to the unique solution $u \in C$ of the equation (1.5).

The first iterative methods for approximating solutions of Hammerstein equations, in real Banach spaces more general than Hilbert spaces, as far as we know, were obtained by Chidume and Zegeye [25] (see also Chidume [10], Chap. 13).

Let $B$ be a real Banach space and $F, K: B \rightarrow B$ be accretive-type mappings. Let $E:=B \times B$. Then, define $T: E \rightarrow E$ by

$$
T[u, v]=[F u-v, K v+u], \quad \text { for }[u, v] \in E .
$$

We note that $T[u, v]=0 \Longleftrightarrow u$ solves (1.1) and $v=F u$. With this, Chidume and Zegeye [25] were able to prove strong convergence of an iterative algorithm defined in the Cartesian product space $E$ to a solution of the Hammerstein equation (1.1). Extensions of these early results of Chidume and Zegeye [25] were obtained by several authors (see, e.g., Chidume and Zegeye [26, 27], Chidume and Djitte [15-17], Chidume and Ofoedu [21], Chidume and Shehu [22, 23], Chidume et al. [12, 20] Djitte and Sene [29], Ofoedu and Onyi [39], Ofoedu and Malonza [38], Shehu [43], Minjibir and Mohammed [34], and the references contained therein).

In 2015, Chidume and Shehu [24] introduced a new explicit iterative algorithm in the setting of a real Hilbert space and proved the following strong convergence theorem.

Theorem 1.2 Let $H$ be a real Hilbert space. Let $K, F: H \rightarrow H$ be bounded, continuous and monotone mappings. Suppose that $u^{*} \in H$ is a solution of $u+K F u=0$. Let $\left\{u^{n}\right\}$ and $\left\{v^{n}\right\}$ be sequences in $H$ defined iteratively from arbitrary $u^{1}, v^{1} \in H$ by

$$
\left\{\begin{array}{l}
u^{n+1}=u^{n}-\beta_{n}^{2}\left(F u^{n}-v^{n}\right)-\beta_{n} \alpha_{n}\left(u^{n}-u^{1}\right), \\
v^{n+1}=v^{n}-\beta_{n}^{2}\left(K v^{n}+u^{n}\right)-\beta_{n} \alpha_{n}\left(v^{n}-v^{1}\right),
\end{array}\right.
$$

where $\left\{\alpha_{n}\right\}$ and $\left\{\beta_{n}\right\}$ are real sequences in $(0,1)$ such that

$$
\sum_{n=1}^{\infty} \beta_{n}^{2}<\infty \quad \text { and } \quad \sum_{n=1}^{\infty} \alpha_{n} \beta_{n}=\infty
$$

Then, there exists a real constant $\epsilon_{0}>0$ such that if $\beta_{n}<\epsilon_{0} \alpha_{n}, \forall n \geq n_{0}$, for some $n_{0} \in \mathbb{N}$, the sequence $\left\{u^{n}\right\}$ converges to $u^{*}$. 
Recently, Chidume and Bello [13], studied a coupled explicit algorithm different from given by (1.7) and proved the following strong convergence theorem in $L_{p}$ spaces, $1<p<2$.

Theorem 1.3 Let $E=L_{p}, 1<p<2$. Let $F: E \rightarrow E^{*}$ and $K: E^{*} \rightarrow E$ be strongly monotone and bounded maps. For $\left(u^{0}, v^{0}\right) \in E \times E^{*}$, define the sequences $\left\{u^{n}\right\}$ and $\left\{v^{n}\right\}$ in $E$ and $E^{*}$ respectively by

$$
\left\{\begin{array}{l}
u^{n+1}=J^{-1}\left(J u^{n}-\alpha_{n}\left(F u^{n}-v^{n}\right)\right), \\
v^{n+1}=J\left(J^{-1} v^{n}-\alpha_{n}\left(K v^{n}+u^{n}\right)\right), \quad n \geq 0,
\end{array}\right.
$$

where $\left\{\alpha_{n}\right\} \subset(0,1)$ satisfies the following conditions: $\sum_{n=1}^{\infty} \alpha_{n}=\infty, \sum_{n=1}^{\infty} \alpha_{n}^{2}<\infty$ and $\sum_{n=1}^{\infty}\left(\alpha_{n}\right)^{\frac{q}{q-1}}<\infty$, with $q$ being such that $\frac{1}{q}+\frac{1}{p}=1$. Assume that the equation $u+K F u=0$ has a solution. Then there exists $\gamma_{0}>0$ such that if $\alpha_{n} \leq \gamma_{0}$ for all $n \geq 1$, the sequences $\left\{u^{n}\right\}$ and $\left\{v^{n}\right\}$ converge strongly to $u$ and $v$, respectively, where $u$ is the solution of $u+K F u=0$ with $v=F u$.

In 2017, Uba et al. [44] proved the following theorem:

Theorem 1.4 Let E be a uniformly convex and uniformly smooth real Banach space and $F: E \rightarrow E^{*}, K: E^{*} \rightarrow E$ be maximal monotone and bounded maps. For $u_{1} \in E, v_{1} \in E^{*}$ define the sequences $\left\{u_{n}\right\}$ and $\left\{v^{n}\right\}$ in $E$ and $E^{*}$, respectively, by

$$
\left\{\begin{array}{l}
u^{n+1}=J^{-1}\left(J u^{n}-\lambda_{n}\left(F u^{n}-v^{n}\right)-\lambda_{n} \theta_{n}\left(J u^{n}-J u^{1}\right)\right), \\
v^{n+1}=J\left(J^{-1} v^{n}-\lambda_{n}\left(K v^{n}+u^{n}\right)-\lambda_{n} \theta_{n}\left(J^{-1} v^{n}-J^{-1} v^{1}\right)\right), \quad n \geq 1 .
\end{array}\right.
$$

Assume that the equation $u+K F u=0$ has a solution. Then, the sequences $\left\{u^{n}\right\}$ and $\left\{v^{n}\right\}$ converge strongly to $u^{*}$ and $v^{*}$, respectively, where $u^{*}$ is the solution of $u+K F u=0$ with $v^{*}=F u^{*}$, where $\left\{\lambda_{n}\right\}$ and $\left\{\theta_{n}\right\}$ are sequences in $(0,1)$ satisfying some appropriate conditions.

Remark 1 We observe that in Theorems 1.2, 1.3, and 1.4, the operators $K$ and $F$ are required to be bounded. This is a serious drawback on these theorems.

It is our purpose in this paper to first prove a significant improvement of Theorem 1.2. We introduce a new recursive formula, and extend Theorem 1.2 to uniformly convex and uniformly smooth real Banach spaces and, at the same time, dispense with the requirement in Theorem 1.2 that the mappings $K$ and $F$ be continuous and bounded. Furthermore, using our new recursive formula, we are able to prove strong convergence without imposing any boundedness assumption on the mappings $K$ and $F$. This makes our theorem much more applicable than Theorem 1.4. Finally, we give numerical experiments to illustrate the convergence of the sequence of our theorem.

\section{Preliminaries}

In this section, we present definitions of some terms, and results that will be needed in the proof of our main theorem. 
Definition 2.1 Let $E$ be a smooth real Banach space. The Lyapunov functional $\phi: E \times E \rightarrow$ $\mathbb{R}$ is defined by

$$
\phi(u, v)=\|u\|^{2}-2\langle u, J v\rangle+\|v\|^{2}, \quad \forall u, v \in E .
$$

It was introduced by Alber and has been studied by many authors (see, e.g., Alber [1]; Chidume et al. [11, 19]; Kamimura and Takahashi [32]; Nilsrakoo and Saejung [37]; and the references therein). It is easy to see that from the definition of $\phi$,

$$
(\|u\|-\|v\|)^{2} \leq \phi(u, v) \leq(\|u\|+\|v\|)^{2}, \quad \forall u, v \in E
$$

Definition 2.2 Let $E$ be a normed linear space and consider the map $V: E \times E^{*} \rightarrow \mathbb{R}$ defined by

$$
V\left(u, u^{*}\right)=\|u\|^{2}-2\left\langle u, u^{*}\right\rangle+\left\|u^{*}\right\|, \quad \forall u \in E, u^{*} \in E^{*} .
$$

Observe that

$$
V\left(u, u^{*}\right)=\phi\left(u, J^{-1} u^{*}\right), \quad \forall u \in E, u^{*} \in E^{*} .
$$

Lemma 2.3 (Alber and Ryazantseva [1]) Let E be a reflexive strictly convex and smooth Banach space with $E^{*}$ as its dual. Then,

$$
V\left(u, u^{*}\right)+2\left(J^{-1} u^{*}-u, v^{*}\right) \leq V\left(u, u^{*}+v^{*}\right),
$$

for all $u \in E$ and $u^{*}, v^{*} \in E^{*}$.

Lemma 2.4 (Alber and Ryazantseva [1]) Let E be a reflexive strictly convex and smooth Banach space with dual space $E^{*}$. Let $W: E \times E \rightarrow \mathbb{R}$ be defined by $W(u, v)=\frac{1}{2} \phi(v, u)$. Then, $\forall u, v, s \in E$,

$$
W(u, v)-W(s, u) \geq\langle s-v, J u-J s\rangle, \quad \text { i.e., } \phi(v, u)-\phi(u, s) \geq 2\langle s-v, J u-J s\rangle,
$$

and also, $W(u, v) \leq\langle u-v, J u-J v\rangle$.

Lemma 2.5 (Chidume and Idu [18]) Let E be a smooth real Banach space with dual space $E^{*}$. Let $\phi: E \times E \rightarrow \mathbb{R}$ be the Lyapunov functional. Then,

$$
\phi(v, u)=\phi(u, v)-\langle u+v, J u-J v\rangle+2\left(\|u\|^{2}-\|v\|^{2}\right), \quad \forall u, v \in E .
$$

Lemma 2.6 (Rockafellar [42]; see also, Pascali and Sburlan [40]) A monotone mapping $T: E \rightarrow 2^{E^{*}}$ is locally bounded at the interior points of its domain.

Remark 2 Let $E^{*}$ be a real strictly convex dual space with a Fréchet differentiable norm. Let $A: E \rightarrow E^{*}$ be a maximal monotone operator with no monotone extension. Let $s \in E^{*}$ be fixed. Then for every $\rho>0$, there exists a unique $u_{\rho} \in E$ such that $J u_{\rho}+\rho A u_{\rho} \ni s$ (see Reich [41]). Setting $J_{\rho} s=u_{\rho}$, we have the resolvent $J_{\rho}:=(J+\rho A)^{-1}: E^{*} \rightarrow E$ of $A$ for every $\rho>0$. The following is a celebrated result of Reich. 
Lemma 2.7 (Reich [41]) Let $E^{*}$ be a real strictly convex dual space with a Fréchet differentiable norm, and let $A$ be a maximal monotone operator from $E$ to $E^{*}$ such that $A^{-1} 0 \neq \emptyset$. Let $s \in E^{*}$ be arbitrary but fixed. For each $\rho>0$ there exists a unique $u_{\rho} \in E$ such that $J u_{\rho}+\rho A u_{\rho} \ni$ s. Furthermore, $u_{\rho}$ converges strongly to a unique point $p \in A^{-1} 0$.

Remark 3 From Lemma 2.7, setting $\rho_{n}=\frac{\beta_{n}}{\alpha_{n}}$ where $\frac{\alpha_{n}}{\beta_{n}} \rightarrow 0$, as $n \rightarrow \infty, s=J v$ for some $v \in E$, and $y^{n}:=\left(J+\frac{\beta_{n}}{\alpha_{n}} A\right)^{-1} s$, we obtain

$$
A y^{n}=\frac{\alpha_{n}}{\beta_{n}}\left(J v-J y^{n}\right),
$$

$y^{n} \rightarrow y^{*} \in A^{-1} 0$, where $A: E \rightarrow E^{*}$ is maximal monotone.

Remark 4 Let $r>0$ such that $\|v\| \leq r,\left\|y^{n}\right\| \leq r$, for all $n \geq 1$. We observe that equation (2.4) yields

$$
J y^{n-1}-J y^{n}+\frac{\beta_{n}}{\alpha_{n}}\left(A y^{n-1}-A y^{n}\right)=\left(\frac{\alpha_{n-1} \beta_{n}-\alpha_{n} \beta_{n-1}}{\alpha_{n} \beta_{n-1}}\right)\left(J v-J y^{n-1}\right) .
$$

From (2.5), applying Cauchy-Schwarz inequality, and using the fact that $A$ is monotone, we obtain

$$
\left\langle y^{n-1}-y^{n}, J y^{n-1}-J y^{n}\right\rangle \leq\left(\frac{\alpha_{n-1} \beta_{n}-\alpha_{n} \beta_{n-1}}{\alpha_{n} \beta_{n-1}}\right)\left\|J v-J y^{n-1}\right\|\left\|y^{n-1}-y^{n}\right\| .
$$

It follows that if $E$ is uniformly convex and uniformly smooth, using a result of Alber and Ryazantseva [1] (see, e.g., Chidume and Idu [18, Lemma 2.3]), we obtain

$$
\begin{aligned}
(2 L)^{-1} \delta_{E}\left(c_{2}^{-1}\left\|y^{n-1}-y^{n}\right\|\right) & \leq\left(\frac{\alpha_{n-1} \beta_{n}-\alpha_{n} \beta_{n-1}}{\alpha_{n} \beta_{n-1}}\right)\left\|J v-J y^{n-1}\right\|\left\|y^{n-1}-y^{n}\right\| \\
& \leq 2 r \sup \left\{\left\|J v-J y^{n-1}\right\|\right\}\left(\frac{\alpha_{n-1} \beta_{n}-\alpha_{n} \beta_{n-1}}{\alpha_{n} \beta_{n-1}}\right),
\end{aligned}
$$

which gives

$$
\left\|y^{n-1}-y^{n}\right\| \leq c_{2} \delta_{E}^{-1}\left(\frac{\alpha_{n-1} \beta_{n}-\alpha_{n} \beta_{n-1}}{\alpha_{n} \beta_{n-1}} D\right) .
$$

Similarly, using a result of Alber and Ryazantseva [1] (see, e.g., Chidume and Idu [18, Lemma 2.4]), we obtain

$$
\left\|J y^{n-1}-J y^{n}\right\| \leq c_{2} \delta_{E^{*}}^{-1}\left(\frac{\alpha_{n-1} \beta_{n}-\alpha_{n} \beta_{n-1}}{\alpha_{n} \beta_{n-1}} D\right) .
$$

Lemma 2.8 (Kamimura and Takahashi [32]) Let E be a uniformly convex and smooth real Banach space, and let $\left\{u^{n}\right\}$ and $\left\{v^{n}\right\}$ be two sequences of E. If either $\left\{u^{n}\right\}$ or $\left\{v^{n}\right\}$ is bounded and $\phi\left(u^{n}, v^{n}\right) \rightarrow 0$ then $\left\|u^{n}-v^{n}\right\| \rightarrow 0$.

Lemma 2.9 (Chidume and Idu [18]) Let $X, Y$ be real uniformly convex and uniformly smooth spaces. Then $E=X \times Y$ is uniformly convex and uniformly smooth. 
Lemma 2.10 (Chidume and Idu [18]) Let E be a uniformly convex and uniformly smooth real Banach space and $F: E \rightarrow E^{*}, K: E^{*} \rightarrow E$ be maximal monotone. Define $A: E \times E^{*} \rightarrow$ $E^{*} \times E$ by

$$
A[u, v]=[F u-v, K v+u], \quad \forall[u, v] \in E \times E^{*} .
$$

Then, $A$ is maximal monotone.

Remark 5 From Lemma 2.7, setting $\rho_{n}:=\frac{\beta_{n}}{\alpha_{n}}$ where $\frac{\alpha_{n}}{\beta_{n}} \rightarrow 0$, as $n \rightarrow \infty, z=\left[z^{1}, z^{2}\right]=$ $J_{E \times E^{*}}\left[u^{1}, v^{1}\right]$, for some $\left[u^{1}, v^{1}\right] \in E \times E^{*}$, and $\left[y^{n},\left(y^{n}\right)^{*}\right]:=\left(J_{E \times E^{*}}+\frac{\beta_{n}}{\alpha_{n}} A\right)^{-1}\left[z^{1}, z^{2}\right]$, we obtain that

$$
\begin{aligned}
& J y^{n}+\frac{\beta_{n}}{\alpha_{n}}\left(F y^{n}-\left(y^{n}\right)^{*}\right)=z^{1}, \quad \forall n \geq 1, \quad \text { and } \\
& J^{-1}\left(y^{n}\right)^{*}+\frac{\beta_{n}}{\alpha_{n}}\left(K\left(y^{n}\right)^{*}+y^{n}\right)=z^{2}, \quad \forall n \geq 1 .
\end{aligned}
$$

Remark 6 Let $y^{n} \rightarrow u^{*}$ and $\left(y^{n}\right)^{*} \rightarrow v^{*}$. From Lemma 2.7 we have that $\left[y^{n},\left(y^{n}\right)^{*}\right]$ converges to a point in $A^{-1} 0$. This implies that $\left[u^{*}, v^{*}\right] \in A^{-1} 0$. Consequently, $A\left[u^{*}, v^{*}\right]=0$, that is, $F u^{*}-v^{*}=0$ and $K v^{*}+u^{*}=0$. Hence, $v^{*}=F u^{*}$ and $u^{*}+K F u^{*}=0$.

The analytical representations of duality maps are known in $L_{p}(\Omega)$ and $L_{q}(\Omega)$ spaces, for $p>1$ and $q>1$ such that $\frac{1}{p}+\frac{1}{q}=1$ :

$$
\begin{aligned}
& J z=\|z\|_{L_{p}}^{2-p}|z(s)|^{p-2} z(s) \in L_{q}(\Omega), \quad s \in \Omega, \\
& J^{-1} z=\|z\|_{L_{q}}^{2-q}|z(s)|^{q-2} z(s) \in L_{p}(\Omega), \quad s \in \Omega
\end{aligned}
$$

(see, e.g., Lindenstrauss and Tzafriri [33]).

\section{Main result}

Definition 3.1 A mapping $A: E \rightarrow E^{*}$ is quasi-bounded if for any $\sigma>0$ there exists $\tau>0$ such that whenever $\langle y, A y\rangle \leq \sigma\|y\|$ and $\|y\| \leq \sigma$ then $\|A y\| \leq \tau$.

We first prove the following lemma (see Pascali and Sburlan [40, Chap. III, Lemma 3.6]).

Lemma 3.2 Let $E$ be a real normed space with dual space $E^{*}$. Any monotone map A: $D(A) \subset E \rightarrow E^{*}$ with $0 \in \operatorname{Int} D(A)$ is quasi-bounded.

Proof By Lemma 2.6, $A$ is locally bounded at 0 . Now, $A$ is locally bounded at $0 \Rightarrow \exists r>0$ such that

$$
\|A x\| \leq \sigma, \quad \forall x \in B(0, r) \text {, for some } \sigma>0 .
$$

Using this $\sigma>0$, suppose $\langle A y, y\rangle \leq \sigma\|y\|$ and $\|y\| \leq \sigma$. Then, by monotonicity of $A$, we have

$$
\langle A y, y\rangle \geq\langle A y, x\rangle+\langle A x, y-x\rangle, \quad \forall x \in B(0, r) .
$$


Observe that

$$
\begin{aligned}
\langle A x, y-x\rangle & \leq|\langle A x, y-x\rangle| \\
& \leq\|A x\|(\|y\|+\|x\|) \leq \sigma(\|y\|+r) .
\end{aligned}
$$

Thus,

$$
\begin{aligned}
\langle A y, x\rangle & \leq\langle A y, y\rangle+\langle A x, x-y\rangle \\
& \leq \sigma\|y\|+\sigma(\|y\|+r), \quad \forall x \in B(0, r) .
\end{aligned}
$$

This implies that

$$
|\langle A y, x\rangle| \leq \sigma\|y\|+\sigma(\|y\|+r), \quad \forall x \in B(0, r) .
$$

Thus,

$$
\sup _{\|x\| \leq r}|\langle A y, x\rangle| \leq \sigma\|y\|+\sigma(\|y\|+r) .
$$

And therefore,

$$
\|A y\| \leq \frac{\sigma}{r}\|y\|+\frac{\sigma}{r}(\|y\|+r),
$$

implying easily the quasi-boundedness of $A$.

In Lemma 3.3 below, the sequences $\left\{\alpha_{n}\right\},\left\{\beta_{n}\right\} \subset(0,1)$ satisfy the following conditions:

(i) $\lim _{n \rightarrow \infty} \alpha_{n}=0, \lim _{n \rightarrow \infty} \frac{\alpha_{n}}{\beta_{n}}=0, \sum_{n=1}^{\infty} \alpha_{n} \beta_{n}=\infty$,

(ii) $\delta_{E}^{-1}\left(\beta_{n} M_{0}\right) \leq \alpha_{n} \gamma_{0} ; \delta_{E^{*}}^{-1}\left(\beta_{n} M_{0}^{*}\right) \leq \alpha_{n} \gamma_{0} ; \beta_{n} S^{*} \leq \alpha_{n} \gamma_{0}, \forall n \geq 1$, for some constants, $M_{0}, M_{0}^{*}, S^{*}, \gamma_{0}>0$.

Lemma 3.3 Let E be a uniformly convex and uniformly smooth real Banach space. Let $F: E \rightarrow E^{*}, K: E^{*} \rightarrow E$ be maximal monotone mappings. For arbitrary $x \in E, y \in E^{*}$, define the sequences $\left\{u^{n}\right\}$ and $\left\{v^{n}\right\}$ in $E$ and $E^{*}$, respectively, for $u^{1} \in E, v^{1} \in E^{*}$ by

$$
\begin{aligned}
& u^{n+1}=J^{-1}\left(J u^{n}-\beta_{n}^{2}\left(F u^{n}-v^{n}\right)-\alpha_{n} \beta_{n}\left(J u^{n}-J x\right)\right), \\
& v^{n+1}=J\left(J^{-1} v^{n}-\beta_{n}^{2}\left(K v^{n}+u^{n}\right)-\alpha_{n} \beta_{n}\left(J^{-1} v^{n}-J^{-1} y\right)\right),
\end{aligned}
$$

where $\left\{\alpha_{n}\right\}$ and $\left\{\beta_{n}\right\}$ are sequences in $(0,1)$ satisfying conditions (i) and (ii). Assume that the equation $u+K F u=0$ has a solution. Then, the sequences $\left\{u^{n}\right\}$ and $\left\{v^{n}\right\}$ are bounded.

Proof Now, to show that sequences $\left\{u^{n}\right\}$ and $\left\{v^{n}\right\}$ are bounded, set

$$
w^{n}=\left(u^{n}, v^{n}\right), \quad w^{*}=\left(u^{*}, v^{*}\right) \in W=E \times E^{*},
$$

where $u^{*}$ is a solution (1.1) with $v^{*}=F u^{*}$. 
Define $\Phi: W \times W \rightarrow \mathbb{R}$ by

$$
\Phi\left(w^{1}, w^{2}\right)=\phi\left(u^{1}, u^{2}\right)+\phi\left(v^{1}, v^{2}\right)
$$

where $w^{1}=\left(u^{1}, v^{1}\right)$ and $w^{2}=\left(u^{2}, v^{2}\right)$. Let $W$ be endowed with the norm

$$
\|(u, v)\|_{W}=\left(\|u\|_{E}^{2}+\|v\|_{E^{*}}^{2}\right)^{\frac{1}{2}} .
$$

It suffices to show that $\left\{w^{n}\right\}$ is bounded. We show this by induction. Let $w^{1} \in W$. Then there exists $r>0$ such that $\Phi\left(w^{*}, w^{1}\right) \leq \frac{r}{8}$. Let $B:=\left\{w=(u, v) \in W: \Phi\left(w^{*}, w\right) \leq r\right\}$. It suffices to show that $\Phi\left(w^{*}, w^{n}\right) \leq r$, for all $n \geq 1$. Let $w, w^{1} \in B$ and $\alpha, \beta \in(0,1)$. Then,

$$
\Phi\left(w^{*}, w\right) \leq r, \quad \text { i.e., } \phi\left(u^{*}, u\right)+\phi\left(v^{*}, v\right) \leq r .
$$

Therefore,

$$
\phi\left(u^{*}, u\right) \leq r \quad \text { and } \quad \phi\left(v^{*}, v\right) \leq r .
$$

Now, using inequality (2.2),

$$
\phi\left(u^{*}, u\right) \leq r \quad \Longrightarrow \quad\|u\| \leq\left\|u^{*}\right\|+\sqrt{r}
$$

Since $F$ is also locally bounded at $u$, there exists $k_{1}>0$ such that

$$
\langle u, F u\rangle \leq k_{1}\|u\|
$$

Define $\sigma:=\max \left\{k_{1},\left\|u^{*}\right\|+\sqrt{r}\right\}$. Hence, $\langle u, F u\rangle \leq \sigma\|u\|$ and $\|u\| \leq \sigma$. By Lemma 3.2, $F$ is quasi-bounded. Thus, there exists $\tau_{1}>0$ such that

$$
\|F u\| \leq \tau_{1}, \quad \forall(u, v) \in B
$$

Similarly, there exists $\tau_{2}>0$ such that

$$
\|K v\| \leq \tau_{2}, \quad \forall(u, v) \in B
$$

Define

$$
S^{*}=\max \left\{\begin{array}{c}
\sup \{\|\beta(F u-v)+\alpha(J u-J x)\|\}+1, \sup \{\|J u-J x\|\}+1, \\
\sup \left\{\left\|J^{-1}\left(J u-\beta^{2}(F u-v)-\alpha \beta(J u-J x)\right)-u\right\|\right\}+1, \\
\sup \left\{\left\|\beta(K v+u)+\alpha\left(J^{-1} v-J^{-1} y\right)\right\|\right\}+1, \sup \left\{\left\|J^{-1} v-J^{-1} y\right\|\right\}+1, \\
\sup \left\{\left\|J\left(J^{-1} v-\beta^{2}(K v+u)-\alpha \beta\left(J^{-1} v-J^{-1} y\right)\right)-v\right\|\right\}+1
\end{array}\right\},
$$

where all the suprema are taken over $u, v \in B$. The boundedness of $K, F, J$ on bounded sets guarantees that $S^{*}$ is well defined. Let $M:=\max \left\{c_{2} S^{*}, S^{*}\right\}, M_{0}=M_{0}^{*}:=2 L\left(S^{*}\right)^{2} \gamma_{0}:=$ $\min \left\{1, \frac{r}{32 M}\right\}$. Then, for $n=1$, by construction $\Phi\left(w^{*}, w^{1}\right) \leq r$. Assume $\Phi\left(w^{*}, w^{n}\right) \leq r$, for some $n \geq 1$, i.e.,

$$
\phi\left(u^{*}, u^{n}\right)+\phi\left(v^{*}, v^{n}\right) \leq r, \quad \text { for some } n \geq 1 \text {. }
$$


We show that $\Phi\left(w^{*}, w^{n+1}\right) \leq r$. For contradiction, suppose $r<\Phi\left(w^{*}, w^{n+1}\right)$. Using a result of Alber and Ryazantseva [1] (see, e.g., Chidume and Idu, [18, Lemma 2.3]) and recurrence relation (3.1), we have

$$
\begin{aligned}
& (2 L)^{-1} \delta_{E}\left(c_{2}^{-1}\left\|u^{n+1}-u^{n}\right\|\right) \\
& \quad \leq\left\langle J u^{n+1}-J u^{n}, u^{n+1}-u^{n}\right\rangle \\
& \quad \leq\left\|J u^{n+1}-J u^{n}\right\|\left\|u^{n+1}-u^{n}\right\| \\
& \quad \leq \beta_{n}\left[\sup \left\{\left\|\beta_{n}\left(F u^{n}-v^{n}\right)+\alpha_{n}\left(J u^{n}-J x\right)\right\|\right\}+1\right]\left\|u^{n+1}-u^{n}\right\| .
\end{aligned}
$$

Thus,

$$
\left\|u^{n+1}-u^{n}\right\| \leq c_{2} \delta_{E}^{-1}\left(\beta_{n} M_{0}\right)
$$

Similarly, using a result of Alber and Ryazantseva [1] (see, e.g., Chidume and Idu, [18, Lemma 2.4]) and recurrence relation (3.2), we obtain

$$
\left\|v^{n+1}-v^{n}\right\| \leq c_{2} \delta_{E^{*}}^{-1}\left(\beta_{n} M_{0}^{*}\right)
$$

Now, using recurrence relation (3.1), Lemma 2.3, and inequality (3.3), we have

$$
\begin{aligned}
& \phi\left(u^{*}, u^{n+1}\right) \\
&= V\left(u^{*}, J u^{n}-\beta_{n}^{2}\left(F u^{n}-v^{n}\right)-\alpha_{n} \beta_{n}\left(J u^{n}-J x\right)\right) \\
& \leq V\left(u^{*}, J u^{n}\right)-2\left\langle u^{n+1}-u^{*}, \beta_{n}^{2}\left(F u^{n}-v^{n}\right)+\alpha_{n} \beta_{n}\left(J u^{n}-J x\right)\right\rangle \\
&= \phi\left(u^{*}, u^{n}\right)-2\left\langle u^{n}-u^{*}, \beta_{n}^{2}\left(F u^{n}-v^{n}\right)+\alpha_{n} \beta_{n}\left(J u^{n}-J x\right)\right\rangle \\
&-2\left\langle u^{n+1}-u^{n}, \beta_{n}^{2}\left(F u^{n}-v^{n}\right)+\alpha_{n} \beta_{n}\left(J u^{n}-J x\right)\right\rangle \\
& \leq \phi\left(u^{*}, u^{n}\right)-2\left\langle u^{n}-u^{*}, \beta_{n}^{2}\left(F u^{n}-v^{n}\right)+\alpha_{n} \beta_{n}\left(J u^{n}-J x\right)\right\rangle \\
&+2 \beta_{n}\left\|u^{n+1}-u^{n}\right\|\left\|\beta_{n}\left(F u^{n}-v^{n}\right)+\alpha_{n}\left(J u^{n}-J x\right)\right\| \\
& \leq \phi\left(u^{*}, u^{n}\right)-2 \beta_{n}^{2}\left\langle u^{n}-u^{*}, F u^{n}-v^{n}\right\rangle-2 \alpha_{n} \beta_{n}\left\langle u^{n}-u^{*}, J u^{n}-J x\right\rangle \\
&+2 \beta_{n} c_{2} S^{*} \delta_{E}^{-1}\left(\beta_{n} M_{0}\right) .
\end{aligned}
$$

Observe that, by monotonicity of $F$ and the fact that $v^{*}=F u^{*}$, we have

$$
\left\langle u^{n}-u^{*}, F u^{n}-v^{n}\right\rangle \geq\left\langle u^{n}-u^{*}, v^{*}-v^{n}\right\rangle .
$$

Thus, substituting this in inequality (3.5), we have

$$
\begin{aligned}
\phi\left(u^{*}, u^{n+1}\right) \leq & \phi\left(u^{*}, u^{n}\right)-2 \beta_{n}^{2}\left\langle u^{n}-u^{*}, v^{*}-v^{n}\right\rangle \\
& -2 \alpha_{n} \beta_{n}\left\langle u^{n}-u^{n+1}, J u^{n}-J x\right\rangle-2 \alpha_{n} \beta_{n}\left\langle u^{n+1}-u^{*}, J u^{n}-J u^{n+1}\right\rangle \\
& -2 \alpha_{n} \beta_{n}\left\langle u^{n+1}-u^{*}, J u^{n+1}-J x\right\rangle+2 \beta_{n} c_{2} S^{*} \delta_{E}^{-1}\left(\beta_{n} M_{0}\right) .
\end{aligned}
$$


Using Lemma 2.4, we have

$$
-2 \alpha_{n} \beta_{n}\left\langle u^{n+1}-u^{*}, J u^{n+1}-J x\right\rangle \leq \alpha_{n} \beta_{n} \phi\left(u^{*}, x\right)-\alpha_{n} \beta_{n} \phi\left(u^{*}, u^{n+1}\right) .
$$

Substituting this into inequality (3.6), we obtain

$$
\begin{aligned}
& \phi\left(u^{*}, u^{n+1}\right) \\
& \leq \phi\left(u^{*}, u^{n}\right)-\alpha_{n} \beta_{n} \phi\left(u^{*}, u^{n+1}\right)+\alpha_{n} \beta_{n} \phi\left(u^{*}, x\right) \\
&-2 \beta_{n}^{2}\left\langle u^{n}-u^{*}, v^{*}-v^{n}\right\rangle-2 \alpha_{n} \beta_{n}\left\langle u^{n}-u^{n+1}, J u^{n}-J x\right\rangle \\
&-2 \alpha_{n} \beta_{n}\left\langle u^{n+1}-u^{*}, J u^{n}-J u^{n+1}\right\rangle+2 \beta_{n} c_{2} S^{*} \delta_{E}^{-1}\left(\beta_{n} M_{0}\right) \\
& \leq \phi\left(u^{*}, u^{n}\right)-\alpha_{n} \beta_{n} \phi\left(u^{*}, u^{n+1}\right)+\alpha_{n} \beta_{n} \phi\left(u^{*}, x\right) \\
&-2 \beta_{n}^{2}\left\langle u^{n}-u^{*}, v^{*}-v^{n}\right\rangle+2 \alpha_{n} \beta_{n}\left\|u^{n}-u^{n+1}\right\|\left\|J u^{n}-J x\right\| \\
&+2 \alpha_{n} \beta_{n}\left\|u^{n+1}-u^{*}\right\|\left\|J u^{n}-J u^{n+1}\right\|+2 \beta_{n} c_{2} S^{*} \delta_{E}^{-1}\left(\beta_{n} M_{0}\right) \\
& \leq \phi\left(u^{*}, u^{n}\right)-\alpha_{n} \beta_{n} \phi\left(u^{*}, u^{n+1}\right)+\alpha_{n} \beta_{n} \phi\left(u^{*}, x\right) \\
&-2 \beta_{n}^{2}\left\langle u^{n}-u^{*}, v^{*}-v^{n}\right\rangle+2 \alpha_{n} \beta_{n} c_{2} S^{*} \delta_{E}^{-1}\left(\beta_{n} M_{0}\right) \\
&+2 \alpha_{n} \beta_{n}\left(\beta_{n} S^{*}\right) S^{*}+2 \beta_{n} c_{2} S^{*} \delta_{E}^{-1}\left(\beta_{n} M_{0}\right) .
\end{aligned}
$$

Similarly, using recurrence relation (3.2), Lemma 2.3, inequality (3.4), monotonicity of $K$, the fact that $K v^{*}=-u^{*}$, and Lemma 2.4, we obtain

$$
\begin{aligned}
\phi\left(v^{*}, v^{n+1}\right) \leq & \phi\left(v^{*}, v^{n}\right)-\alpha_{n} \beta_{n} \phi\left(v^{*}, v^{n+1}\right)+\alpha_{n} \beta_{n} \phi\left(v^{*}, y\right) \\
& -2 \beta_{n}^{2}\left\langle v^{n}-v^{*}, u^{n}-u^{*}\right\rangle+2 \alpha_{n} \beta_{n} c_{2} S^{*} \delta_{E^{*}}^{-1}\left(\beta_{n} M_{0}^{*}\right) \\
& +2 \alpha_{n} \beta_{n}\left(\beta_{n} S^{*}\right) S^{*}+2 \beta_{n} c_{2} S^{*} \delta_{E^{*}}^{-1}\left(\beta_{n} M_{0}^{*}\right) .
\end{aligned}
$$

Thus, adding inequalities (3.7) and (3.8), we obtain

$$
\begin{aligned}
r< & \Phi\left(w^{*}, w^{n+1}\right) \\
= & \phi\left(u^{*}, u^{n+1}\right)+\phi\left(v^{*}, v^{n+1}\right) \\
\leq & \Phi\left(w^{*}, w^{n}\right)-\alpha_{n} \beta_{n} \Phi\left(w^{*}, w^{n+1}\right)+\alpha_{n} \beta_{n} \Phi\left(w^{*}, w^{1}\right) \\
& +2 \alpha_{n} \beta_{n} c_{2} S^{*} \delta_{E}^{-1}\left(\beta_{n} M_{0}\right)+2 \alpha_{n} \beta_{n}\left(\beta_{n} S^{*}\right) S^{*} \\
& +2 \beta_{n} c_{2} S^{*} \delta_{E}^{-1}\left(\beta_{n} M_{0}\right)+2 \alpha_{n} \beta_{n} c_{2} S^{*} \delta_{E^{*}}^{-1}\left(\beta_{n} M_{0}^{*}\right) \\
& +2 \alpha_{n} \beta_{n}\left(\beta_{n} S^{*}\right) S^{*}+2 \beta_{n} c_{2} S^{*} \delta_{E^{*}}^{-1}\left(\beta_{n} M_{0}^{*}\right) \\
\leq & \Phi\left(w^{*}, w^{n}\right)-\alpha_{n} \beta_{n} \Phi\left(w^{*}, w^{n+1}\right)+\alpha_{n} \beta_{n} \Phi\left(w^{*}, w^{1}\right) \\
& +2 \alpha_{n}^{2} \beta_{n} c_{2} S^{*} \gamma_{0}+2 \alpha_{n}^{2} \beta_{n} S^{*} \gamma_{0}+2 \alpha_{n} \beta_{n} c_{2} S^{*} \gamma_{0} \\
& +2 \alpha_{n}^{2} \beta_{n} c_{2} S^{*} \gamma_{0}+2 \alpha_{n}^{2} \beta_{n} S^{*} \gamma_{0}+2 \alpha_{n} \beta_{n} c_{2} S^{*} \gamma_{0} \\
\leq & \Phi\left(w^{*}, w^{n}\right)-\alpha_{n} \beta_{n} \Phi\left(w^{*}, w^{n+1}\right)+\alpha_{n} \beta_{n} \Phi\left(w^{*}, w^{1}\right) \\
& +2 \alpha_{n} \beta_{n} M \gamma_{0}+2 \alpha_{n} \beta_{n} M \gamma_{0}+2 \alpha_{n} \beta_{n} M \gamma_{0}
\end{aligned}
$$




$$
\begin{aligned}
& +2 \alpha_{n} \beta_{n} M \gamma_{0}+2 \alpha_{n} \beta_{n} M \gamma_{0}+2 \alpha_{n} \beta_{n} M \gamma_{0} \\
\leq & \Phi\left(w^{*}, w^{n}\right)-\alpha_{n} \beta_{n} \Phi\left(w^{*}, w^{n+1}\right)+\frac{r \alpha_{n} \beta_{n}}{8}+\frac{3 r \alpha_{n} \beta_{n}}{8} \\
\leq & r-r \alpha_{n} \beta_{n}+\frac{r \alpha_{n} \beta_{n}}{2}=r-\frac{r \alpha_{n} \beta_{n}}{2}<r .
\end{aligned}
$$

This is a contradiction. Hence, $\Phi\left(w^{*}, w^{n+1}\right) \leq r$. Thus, $\Phi\left(w^{*}, w^{n}\right) \leq r$, for all $n \geq 1$. Consequently, we have $\phi\left(u^{*}, u^{n}\right) \leq r$ and $\phi\left(v^{*}, v^{n}\right) \leq r$, for all $n \geq 1$. Therefore, using inequality (2.2), we deduce that $\left\{u^{n}\right\}$ and $\left\{v^{n}\right\}$ are bounded.

In Theorem 3.4 below, the sequences $\left\{\alpha_{n}\right\},\left\{\beta_{n}\right\} \subset(0,1)$ satisfy the following conditions:

(i) $\lim _{n \rightarrow \infty} \alpha_{n}=0, \lim _{n \rightarrow \infty} \frac{\alpha_{n}}{\beta_{n}}=0, \sum_{n=1}^{\infty} \alpha_{n} \beta_{n}=\infty$,

(ii) $\delta_{E}^{-1}\left(\beta_{n} M_{0}\right) \leq \alpha_{n} \gamma_{0} ; \delta_{E^{*}}^{-1}\left(\beta_{n} M_{0}^{*}\right) \leq \alpha_{n} \gamma_{0} ; \beta_{n} S^{*} \leq \alpha_{n} \gamma_{0}, \forall n \geq 1$, for some constants, $M_{0}, M_{0}^{*}, S^{*}, \gamma_{0}>0$

(iii) $\delta_{E}^{-1}\left(\eta_{n}\right) \rightarrow 0 ; \delta_{E^{*}}^{-1}\left(\eta_{n}\right) \rightarrow 0$,

(iv) $\frac{\delta_{E}^{-1}\left(\eta_{n}\right)}{\alpha_{n} \beta_{n}} \rightarrow 0 ; \frac{\delta_{E^{*}}^{-1}\left(\eta_{n}\right)}{\alpha_{n} \beta_{n}} \rightarrow 0$, $\eta_{n}=\frac{\alpha_{n-1} \beta_{n}-\alpha_{n} \beta_{n-1}}{\alpha_{n} \beta_{n-1}}$.

Theorem 3.4 Let E be a uniformly convex and uniformly smooth real Banach space. Let $F: E \rightarrow E^{*}, K: E^{*} \rightarrow E$ be maximal monotone mappings. For arbitrary $x \in E, y \in E^{*}$, define the sequences $\left\{u^{n}\right\}$ and $\left\{v^{n}\right\}$ in $E$ and $E^{*}$, respectively, for $u^{1} \in E, v^{1} \in E^{*}$, by

$$
\left\{\begin{array}{l}
u^{n+1}=J^{-1}\left(J u^{n}-\beta_{n}^{2}\left(F u^{n}-v^{n}\right)-\alpha_{n} \beta_{n}\left(J u^{n}-J x\right)\right), \\
v^{n+1}=J\left(J^{-1} v^{n}-\beta_{n}^{2}\left(K v^{n}+u^{n}\right)-\alpha_{n} \beta_{n}\left(J^{-1} v^{n}-J^{-1} y\right)\right),
\end{array}\right.
$$

where $\left\{\alpha_{n}\right\}$ and $\left\{\beta_{n}\right\}$ are sequences in $(0,1)$ satisfying conditions (i)-(iv). Assume that the equation $u+K F u=0$ has a solution. Then, the sequences $\left\{u^{n}\right\}$ and $\left\{v^{n}\right\}$ converge strongly to $u^{*}$ and $v^{*}$, respectively, where $u^{*}$ is a solution of $u+K F u=0$ with $v^{*}=F u^{*}$.

Proof Using Lemmas 2.3 and 2.5, we have

$$
\begin{aligned}
\phi\left(y^{n}, u^{n+1}\right)= & V\left(y^{n}, J u^{n}-\beta_{n}^{2}\left(F u^{n}-v^{n}\right)-\alpha_{n} \beta_{n}\left(J u^{n}-J x\right)\right) \\
\leq & V\left(y^{n}, J u^{n}\right)-2\left\langle u^{n+1}-y^{n}, \beta_{n}^{2}\left(F u^{n}-v^{n}\right)+\alpha_{n} \beta_{n}\left(J u^{n}-J x\right)\right\rangle \\
= & \phi\left(y^{n}, u^{n}\right)-2 \beta_{n}^{2}\left\langle u^{n+1}-y^{n}, F u^{n}-v^{n}\right\rangle-2 \alpha_{n} \beta_{n}\left\langle u^{n+1}-y^{n}, J u^{n}-J x\right\rangle \\
= & \phi\left(u^{n}, y^{n}\right)-2\left\langle u^{n}+y^{n}, J u^{n}-J y^{n}\right\rangle+2\left(\left\|u^{n}\right\|^{2}-\left\|y^{n}\right\|^{2}\right) \\
& -2 \beta_{n}^{2}\left\langle u^{n+1}-y^{n}, F u^{n}-v^{n}\right\rangle-2 \alpha_{n} \beta_{n}\left\langle u^{n+1}-y^{n}, J u^{n}-J x\right\rangle .
\end{aligned}
$$

Observe that

$$
\begin{aligned}
\phi\left(u^{n}, y^{n}\right) & =V\left(u^{n}, J y^{n}\right)=V\left(u^{n}, J y^{n-1}+J y^{n}-J y^{n-1}\right) \\
& \leq V\left(u^{n}, J y^{n-1}\right)-2\left\langle y^{n}-u^{n}, J y^{n-1}-J y^{n}\right\rangle .
\end{aligned}
$$

Thus, substituting this into inequality (3.10) and using Lemma 2.5, we obtain

$$
\phi\left(y^{n}, u^{n+1}\right) \leq V\left(u^{n}, J y^{n-1}\right)-2\left\langle y^{n}-u^{n}, J y^{n-1}-J y^{n}\right\rangle
$$




$$
\begin{aligned}
& -2\left\langle u^{n}+y^{n}, J u^{n}-J y^{n}\right\rangle+2\left(\left\|u^{n}\right\|^{2}-\left\|y^{n}\right\|^{2}\right) \\
& -2 \beta_{n}^{2}\left\langle u^{n+1}-y^{n}, F u^{n}-v^{n}\right\rangle-2 \alpha_{n} \beta_{n}\left\langle u^{n+1}-y^{n}, J u^{n}-J x\right\rangle \\
= & \phi\left(y^{n-1}, u^{n}\right)-2\left\langle y^{n-1}+u^{n}, J y^{n-1}-J u^{n}\right\rangle+2\left(\left\|y^{n-1}\right\|^{2}-\left\|u^{n}\right\|^{2}\right) \\
& -2\left\langle y^{n}-u^{n}, J y^{n-1}-J y^{n}\right\rangle-2\left\langle u^{n}+y^{n}, J u^{n}-J y^{n}\right\rangle+2\left(\left\|u^{n}\right\|^{2}-\left\|y^{n}\right\|^{2}\right) \\
& -2 \beta_{n}^{2}\left\langle u^{n+1}-y^{n}, F u^{n}-v^{n}\right\rangle-2 \alpha_{n} \beta_{n}\left\langle u^{n+1}-y^{n}, J u^{n}-J x\right\rangle \\
= & \phi\left(y^{n-1}, u^{n}\right)+2\left(\left\|y^{n-1}\right\|^{2}-\left\|y^{n}\right\|^{2}\right)+2\left\langle y^{n-1}+u^{n}, J u^{n}-J y^{n-1}\right\rangle \\
& -2\left\langle y^{n}-u^{n}, J y^{n-1}-J y^{n}\right\rangle-2\left\langle u^{n}+y^{n}, J u^{n}-J y^{n}\right\rangle \\
& -2 \beta_{n}^{2}\left\langle u^{n+1}-y^{n}, F u^{n}-v^{n}\right\rangle-2 \alpha_{n} \beta_{n}\left\langle u^{n+1}-y^{n}, J u^{n}-J x\right\rangle .
\end{aligned}
$$

Now, we estimate the boxed term using Lemma 2.4 as

$$
\begin{aligned}
-2 \alpha_{n} & \beta_{n}\left\langle u^{n+1}-y^{n}, J u^{n}-J x\right\rangle \\
= & -2 \alpha_{n} \beta_{n}\left\langle u^{n+1}-u^{n}, J u^{n}-J x\right\rangle-2 \alpha_{n} \beta_{n}\left\langle u^{n}-y^{n-1}, J u^{n}-J y^{n-1}\right\rangle \\
& -2 \alpha_{n} \beta_{n}\left\langle u^{n}-y^{n-1}, J y^{n-1}-J x\right\rangle-2 \alpha_{n} \beta_{n}\left\langle y^{n-1}-y^{n}, J u^{n}-J x\right\rangle \\
\leq & -2 \alpha_{n} \beta_{n}\left\langle u^{n+1}-u^{n}, J u^{n}-J x\right\rangle-\alpha_{n} \beta_{n} \phi\left(y^{n-1}, u^{n}\right) \\
& -2 \alpha_{n} \beta_{n}\left\langle u^{n}-y^{n-1}, J y^{n-1}-J x\right\rangle-2 \alpha_{n} \beta_{n}\left\langle y^{n-1}-y^{n}, J u^{n}-J x\right\rangle .
\end{aligned}
$$

Therefore, substituting this into inequality (3.11), we have

$$
\begin{aligned}
\phi\left(y^{n},\right. & \left.u^{n+1}\right) \\
\leq & \phi\left(y^{n-1}, u^{n}\right)+2\left(\left\|y^{n-1}\right\|^{2}-\left\|y^{n}\right\|^{2}\right)+2\left\langle y^{n-1}+u^{n}, J u^{n}-J y^{n-1}\right\rangle \\
& -2\left\langle y^{n}-u^{n}, J y^{n-1}-J y^{n}\right\rangle-2\left\langle u^{n}+y^{n}, J u^{n}-J y^{n}\right\rangle \\
& -2 \beta_{n}^{2}\left\langle u^{n+1}-y^{n}, F u^{n}-v^{n}\right\rangle-2 \alpha_{n} \beta_{n}\left\langle u^{n+1}-u^{n}, J u^{n}-J x\right\rangle \\
& -\alpha_{n} \beta_{n} \phi\left(y^{n-1}, u^{n}\right)-2 \alpha_{n} \beta_{n}\left\langle u^{n}-y^{n-1}, J y^{n-1}-J x\right\rangle \\
& -2 \alpha_{n} \beta_{n}\left\langle y^{n-1}-y^{n}, J u^{n}-J x\right\rangle \\
= & \phi\left(y^{n-1}, u^{n}\right)+2\left(\left\|y^{n-1}\right\|^{2}-\left\|y^{n}\right\|^{2}\right)+2\left\langle y^{n-1}-y^{n}, J u^{n}-J y^{n-1}\right\rangle \\
& +2\left\langle u^{n}+y^{n}, J u^{n}-J y^{n-1}\right\rangle-2\left\langle y^{n}-u^{n}, J y^{n-1}-J y^{n}\right\rangle \\
& -2\left\langle u^{n}+y^{n}, J u^{n}-J y^{n}\right\rangle-2 \beta_{n}^{2}\left\langle u^{n+1}-y^{n}, F u^{n}-v^{n}\right\rangle \\
& -2 \alpha_{n} \beta_{n}\left\langle u^{n+1}-u^{n}, J u^{n}-J x\right\rangle-\alpha_{n} \beta_{n} \phi\left(y^{n-1}, u^{n}\right) \\
& -2 \alpha_{n} \beta_{n}\left\langle u^{n}-y^{n-1}, J y^{n-1}-J x\right\rangle-2 \alpha_{n} \beta_{n}\left\langle y^{n-1}-y^{n}, J u^{n}-J x\right\rangle \\
= & \phi\left(y^{n-1}, u^{n}\right)-\alpha_{n} \beta_{n} \phi\left(y^{n-1}, u^{n}\right)+2\left(\left\|y^{n-1}\right\|^{2}-\left\|y^{n}\right\|^{2}\right) \\
& +2\left\langle y^{n-1}-y^{n}, J u^{n}-J y^{n-1}\right\rangle-2\left\langle u^{n}+y^{n}, J y^{n-1}-J y^{n}\right\rangle \\
& -2\left\langle y^{n}-u^{n}, J y^{n-1}-J y^{n}\right\rangle-2 \beta_{n}^{2}\left\langle u^{n+1}-y^{n}, F u^{n}-v^{n}\right\rangle \\
& -2 \alpha_{n} \beta_{n}\left\langle u^{n+1}-u^{n}, J u^{n}-J x\right\rangle-2 \alpha_{n} \beta_{n}\left\langle u^{n}-y^{n-1}, J y^{n-1}-J x\right\rangle
\end{aligned}
$$




$$
-2 \alpha_{n} \beta_{n}\left\langle y^{n-1}-y^{n}, J u^{n}-J x\right\rangle
$$

We now estimate the boxed terms. Using equation (2.9) and the fact that $F$ is monotone, we obtain

$$
\begin{aligned}
&-2 \beta_{n}^{2}\left\langle u^{n+1}-y^{n}, F u^{n}-v^{n}\right\rangle-2 \alpha_{n} \beta_{n}\left\langle u^{n}-y^{n-1}, J y^{n-1}-J x\right\rangle \\
&=-2 \beta_{n}^{2}\left\langle u^{n+1}-u^{n}, F u^{n}-v^{n}\right\rangle-2 \beta_{n}^{2}\left\langle u^{n}-y^{n}, F u^{n}-v^{n}\right\rangle \\
&-2 \alpha_{n} \beta_{n}\left\langle y^{n}-y^{n-1}, J y^{n-1}-J x\right\rangle-2 \alpha_{n} \beta_{n}\left\langle u^{n}-y^{n}, J y^{n-1}-J y^{n}\right\rangle \\
&+2 \beta_{n}\left\langle u^{n}-y^{n},-\alpha_{n}\left(J y^{n}-J x\right)\right\rangle \\
&=-2 \beta_{n}^{2}\left\langle u^{n+1}-u^{n}, F u^{n}-v^{n}\right\rangle+2 \beta_{n}^{2}\left\langle u^{n}-y^{n}, v^{n}-F u^{n}\right\rangle \\
&-2 \alpha_{n} \beta_{n}\left\langle y^{n}-y^{n-1}, J y^{n-1}-J x\right\rangle-2 \alpha_{n} \beta_{n}\left\langle u^{n}-y^{n}, J y^{n-1}-J y^{n}\right\rangle \\
&+2 \beta_{n}^{2}\left\langle u^{n}-y^{n}, F y^{n}-\left(y^{n}\right)^{*}\right\rangle \\
& \leq-2 \beta_{n}^{2}\left\langle u^{n+1}-u^{n}, F u^{n}-v^{n}\right\rangle-2 \alpha_{n} \beta_{n}\left\langle u^{n}-y^{n}, J y^{n-1}-J y^{n}\right\rangle \\
&-2 \alpha_{n} \beta_{n}\left\langle y^{n}-y^{n-1}, J y^{n-1}-J x\right\rangle+2 \beta_{n}^{2}\left\langle u^{n}-y^{n}, v^{n}-\left(y^{n}\right)^{*}\right\rangle .
\end{aligned}
$$

Thus, substituting this into inequality (3.12), and using inequalities (2.7), (2.8), and (3.3), we obtain

$$
\begin{aligned}
& \phi\left(y^{n},\right.\left.u^{n+1}\right) \\
& \leq \phi\left(y^{n-1}, u^{n}\right)-\alpha_{n} \beta_{n} \phi\left(y^{n-1}, u^{n}\right)+2\left(\left\|y^{n-1}\right\|^{2}-\left\|y^{n}\right\|^{2}\right) \\
&+2\left\langle y^{n-1}-y^{n}, J u^{n}-J y^{n-1}\right\rangle-2\left\langle u^{n}+y^{n}, J y^{n-1}-J y^{n}\right\rangle \\
&-2\left\langle y^{n}-u^{n}, J y^{n-1}-J y^{n}\right\rangle-2 \alpha_{n} \beta_{n}\left\langle u^{n+1}-u^{n}, J u^{n}-J x\right\rangle \\
&-2 \alpha_{n} \beta_{n}\left\langle y^{n-1}-y^{n}, J u^{n}-J x\right\rangle-2 \beta_{n}^{2}\left\langle u^{n+1}-u^{n}, F u^{n}-v^{n}\right\rangle \\
&-2 \alpha_{n} \beta_{n}\left\langle u^{n}-y^{n}, J y^{n-1}-J y^{n}\right\rangle-2 \alpha_{n} \beta_{n}\left\langle y^{n}-y^{n-1}, J y^{n-1}-J x\right\rangle \\
&+2 \beta_{n}^{2}\left\langle u^{n}-y^{n}, v^{n}-\left(y^{n}\right)^{*}\right\rangle \\
& \leq\left(1-\alpha_{n} \beta_{n}\right) \phi\left(y^{n-1}, u^{n}\right)+2 N_{1}\left(\left\|y^{n-1}-y^{n}\right\|+\left\|J y^{n-1}-J y^{n}\right\|\right) \\
&+2 \alpha_{n} \beta_{n} N_{2}\left(\left\|u^{n+1}-u^{n}\right\|+\left\|y^{n-1}-y^{n}\right\|+\left\|J y^{n-1}-J y^{n}\right\|\right) \\
&+2 \beta_{n}^{2} N_{3}\left\|u^{n+1}-u^{n}\right\|+2 \beta_{n}^{2}\left\langle u^{n}-y^{n}, v^{n}-\left(y^{n}\right)^{*}\right\rangle \\
& \leq\left(1-\alpha_{n} \beta_{n}\right) \phi\left(y^{n-1}, u^{n}\right)+2 N_{1}\left(c_{2} \delta_{E}^{-1} \eta_{n}+c_{2} \delta_{E^{*}}^{-1} \eta_{n}\right) \\
&+2 \alpha_{n} \beta_{n} N_{2}\left(c_{2} \delta_{E}^{-1}\left(\beta_{n} M_{0}\right)+c_{2} \delta_{E}^{-1} \eta_{n}+c_{2} \delta_{E^{*}}^{-1} \eta_{n}\right) \\
&+2 \beta_{n}^{2} N_{3} c_{2} \delta_{E}^{-1}\left(\beta_{n} M_{0}\right)+2 \beta_{n}^{2}\left\langle u^{n}-y^{n}, v^{n}-\left(y^{n}\right)^{*}\right\rangle \\
& \leq\left(1-\alpha_{n} \beta_{n}\right) \phi\left(y^{n-1}, u^{n}\right)+\alpha_{n} \beta_{n} \widehat{N}\left(c_{2} \delta_{E}^{-1}\left(\beta_{n} M_{0}\right)+c_{2} \delta_{E}^{-1} \eta_{n}+c_{2} \delta_{E^{*}}^{-1} \eta_{n}\right. \\
&\left.+\beta_{n} c_{2} \gamma_{0}+c_{2} \delta_{E}^{-1}\left(\frac{\eta_{n}}{\alpha_{n} \beta_{n}}\right)+c_{2} \delta_{E^{*}}^{-1}\left(\frac{\eta_{n}}{\alpha_{n} \beta_{n}}\right)\right) \\
&+2 \beta_{n}^{2}\left\langle u^{n}-y^{n}, v^{n}-\left(y^{n}\right)^{*}\right\rangle \\
&
\end{aligned}
$$

for some $N_{1}, N_{2}, N_{3}>0$, and $\widehat{N}=\max \left\{N_{1}, N_{2}, N_{3}\right\}$. 
Similarly, using Lemmas 2.3, 2.5 and 2.4, as well as equation (2.10), we obtain

$$
\begin{aligned}
& \phi\left(\left(y^{n}\right)^{*}, v^{n+1}\right) \\
& \leq \phi\left(\left(y^{n-1}\right)^{*}, v^{n}\right)-\alpha_{n} \beta_{n} \phi\left(\left(y^{n-1}\right)^{*}, v^{n}\right)+2\left(\left\|\left(y^{n-1}\right)^{*}\right\|^{2}-\left\|\left(y^{n}\right)^{*}\right\|^{2}\right) \\
&+2\left\langle\left(y^{n-1}\right)^{*}-\left(y^{n}\right)^{*}, J^{-1} v^{n}-J^{-1} y^{n-1}\right\rangle+2\left\langle\left(y^{n}\right)^{*}+v^{n}, J^{-1} y^{n}-J^{-1}\left(y^{n-1}\right)^{*}\right\rangle \\
&-2\left\langle\left(y^{n}\right)^{*}-v^{n}, J^{-1}\left(y^{n-1}\right)^{*}-J^{-1}\left(y^{n}\right)^{*}\right\rangle-2 \alpha_{n} \beta_{n}\left\langle v^{n+1}-v^{n}, J^{-1} v^{n}-J^{-1} y\right\rangle \\
&-2 \alpha_{n} \beta_{n}\left\langle\left(y^{n-1}\right)^{*}-\left(y^{n}\right)^{*}, J^{-1} v^{n}-J^{-1} y\right\rangle-2 \beta_{n}^{2}\left\langle v^{n+1}-v^{n}, K v^{n}+u^{n}\right\rangle \\
&-2 \alpha_{n} \beta_{n}\left\langle v^{n}-\left(y^{n}\right)^{*}, J^{-1} y^{n-1}-J^{-1}\left(y^{n}\right)^{*}\right\rangle \\
&-2 \alpha_{n} \beta_{n}\left\langle\left(y^{n-1}\right)^{*}-\left(y^{n}\right)^{*}, J^{-1} y^{n-1}-J^{-1} y\right\rangle+2 \beta_{n}^{2}\left\langle v^{n}-\left(y^{n}\right)^{*}, y^{n}-u^{n}\right\rangle .
\end{aligned}
$$

Since $E^{*}$ is uniformly convex and uniformly smooth, using inequalities (2.7), (2.8), and (3.4), we obtain that, for some $\widehat{N}^{*}>0$,

$$
\begin{aligned}
& \phi\left(\left(y^{n}\right)^{*}, v^{n+1}\right) \\
& \leq\left(1-\alpha_{n} \beta_{n}\right) \phi\left(\left(y^{n-1}\right)^{*}, v^{n}\right)+\alpha_{n} \beta_{n} \widehat{N}^{*}\left(c_{2} \delta_{E^{*}}^{-1}\left(\beta_{n} M_{0}^{*}\right)+2 c_{2} \delta_{E}^{-1} \eta_{n}\right. \\
&\left.+\beta_{n} c_{2} \gamma_{0}+c_{2} \delta_{E^{*}}^{-1}\left(\frac{\eta_{n}}{2 \alpha_{n} \beta_{n}}\right)+c_{2} \delta_{E}^{-1}\left(\frac{\eta_{n}}{\alpha_{n} \beta_{n}}\right)\right) \\
&+2 \beta_{n}^{2}\left\langle v^{n}-\left(y^{n}\right)^{*}, y^{n}-u^{n}\right\rangle .
\end{aligned}
$$

Let $p^{n}=\left(y^{n},\left(y^{n}\right)^{*}\right), w^{n}=\left(u^{n}, v^{n}\right)$. Adding inequalities (3.13) and (3.14), we obtain

$$
\begin{aligned}
& \Phi\left(p^{n}, w^{n+1}\right) \\
& \leq\left(1-\alpha_{n} \beta_{n}\right) \Phi\left(p^{n-1}, w^{n}\right)+\alpha_{n} \beta_{n} N\left(c_{2} \delta_{E}^{-1}\left(\beta_{n} M_{0}\right)+2 \beta_{n} c_{2} \gamma_{0}+2 c_{2} \delta_{E}^{-1} \eta_{n}\right. \\
& \quad+2 c_{2} \delta_{E^{*}}^{-1} \eta_{n}+2 c_{2} \delta_{E}^{-1}\left(\frac{\eta_{n}}{\alpha_{n} \beta_{n}}\right)+2 c_{2} \delta_{E^{*}}^{-1}\left(\frac{\eta_{n}}{\alpha_{n} \beta_{n}}\right) \\
& \left.\quad+c_{2} \delta_{E^{*}}^{-1}\left(\beta_{n} M_{0}^{*}\right)\right)
\end{aligned}
$$

where $N=\max \left\{\widehat{N}, \widehat{N}^{*}\right\}$. Now, setting

$$
a_{n}:=\Phi\left(p^{n-1}, w^{n}\right), \quad \sigma_{n}:=\alpha_{n} \beta_{n}, \quad c_{n} \equiv 0,
$$

and

$$
\begin{aligned}
b_{n}:= & \left(c_{2} \delta_{E}^{-1}\left(\beta_{n} M_{0}\right)+2 \beta_{n} c_{2} \gamma_{0}+2 c_{2} \delta_{E}^{-1} \eta_{n}+2 c_{2} \delta_{E^{*}}^{-1} \eta_{n}+2 c_{2} \delta_{E}^{-1}\left(\frac{\eta_{n}}{\alpha_{n} \beta_{n}}\right)\right. \\
& \left.+2 c_{2} \delta_{E^{*}}^{-1}\left(\frac{\eta_{n}}{\alpha_{n} \beta_{n}}\right)+c_{2} \delta_{E^{*}}^{-1}\left(\beta_{n} M_{0}^{*}\right)\right)
\end{aligned}
$$

inequality (3.15) becomes

$$
a_{n+1} \leq\left(1-\sigma_{n}\right) a_{n}+\sigma_{n} b_{n}+c_{n}, \quad n \geq 1 .
$$


It follows from a well known result of Xu [45] that $a_{n} \rightarrow 0$ as $n \rightarrow \infty$, which implies that $\Phi\left(p^{n-1}, w^{n}\right) \rightarrow 0$, as $n \rightarrow \infty$. By Lemma 2.8 , we have $\left\|w^{n}-p^{n-1}\right\|_{W} \rightarrow 0$. Consequently, $\left\|u^{n}-y^{n-1}\right\| \rightarrow 0$. Furthermore, using Remark 6 , since $\left[y^{n},\left(y^{n}\right)^{*}\right] \rightarrow\left[u^{*}, v^{*}\right] \in A^{-1} 0$, we have that $\left\{u^{n}\right\}$ converges strongly to a solution of the Hammerstein equation (1.1) with $v^{*}=F u^{*}$. This completes the proof.

Corollary 3.5 Let $E=L_{p}\left(\right.$ or $\left.W_{p}^{m}(\Omega)\right), 1<p<\infty$. Let $F: E \rightarrow E^{*}, K: E^{*} \rightarrow E$ be maximal monotone mappings. For arbitrary $x \in E, y \in E^{*}$, define the sequences $\left\{u^{n}\right\}$ and $\left\{v^{n}\right\}$ in $E$ and $E^{*}$, respectively, for $u^{1} \in E, v^{1} \in E^{*}$, by

$$
\begin{aligned}
& u^{n+1}=J^{-1}\left(J u^{n}-\beta_{n}^{2}\left(F u^{n}-v^{n}\right)-\alpha_{n} \beta_{n}\left(J u^{n}-J x\right)\right), \\
& v^{n+1}=J\left(J^{-1} v^{n}-\beta_{n}^{2}\left(K v^{n}+u^{n}\right)-\alpha_{n} \beta_{n}\left(J^{-1} v^{n}-J^{-1} y\right)\right),
\end{aligned}
$$

where $\left\{\alpha_{n}\right\}$ and $\left\{\beta_{n}\right\}$ are sequences in $(0,1)$ satisfying conditions (i)-(iv). Assume that the equation $u+K F u=0$ has a solution. Then, the sequences $\left\{u^{n}\right\}$ and $\left\{v^{n}\right\}$ converge strongly to $u^{*}$ and $v^{*}$, respectively, where $u^{*}$ is a solution of $u+K F u=0$ with $v^{*}=F u^{*}$.

Proof $L_{p}$ (or $\left.W_{p}^{m}(\Omega)\right), 1<p<\infty$ are uniformly convex and uniformly smooth. Hence, the conclusion follows from Theorem 3.4.

Corollary 3.6 Let $H$ be a real Hilbert space. Let $F: H \rightarrow H, K: H \rightarrow H$ be maximal monotone mappings. For arbitrary $x, y \in H$, define the sequences $\left\{u^{n}\right\}$ and $\left\{v^{n}\right\}$ in $H$, by: $u^{1}, v^{1} \in H$,

$$
\begin{aligned}
& u^{n+1}=u^{n}-\beta_{n}^{2}\left(F u^{n}-v^{n}\right)-\alpha_{n} \beta_{n}\left(u^{n}-x\right), \\
& v^{n+1}=v^{n}-\beta_{n}^{2}\left(K v^{n}+u^{n}\right)-\alpha_{n} \beta_{n}\left(v^{n}-y\right),
\end{aligned}
$$

where $\left\{\alpha_{n}\right\}$ and $\left\{\beta_{n}\right\}$ are sequences in $(0,1)$ satisfying conditions (i)-(iv). Assume that the equation $u+K F u=0$ has a solution. Then, the sequences $\left\{u^{n}\right\}$ and $\left\{v^{n}\right\}$ converge strongly to $u^{*}$ and $v^{*}$, respectively, where $u^{*}$ is a solution of $u+K F u=0$ with $v^{*}=F u^{*}$.

Remark 7 If $E=L_{p}(1<p<\infty)$, real sequences that satisfy the hypothesis of the theorem and corollaries above are $\alpha_{n}=(n+1)^{-a}$ and $\beta_{n}=(n+1)^{-b}$ with $0<b<\frac{1}{r} a$ and $a+b<\frac{1}{r}$, where $r=\max \{p, q\}$. In particular, without loss of generality, let $r=p$. Then, one can choose $a:=\frac{1}{p+1}$ and $b:=\min \left\{\frac{1}{2 D}, \frac{1}{2 p(p+1)}\right\}$, where $D$ is the constant appearing in Remark 4 . The verifications basically follow as in Chidume and Idu [18]. However, for completeness, we verify conditions (ii)-(iv) of our theorem for $L_{p}$ spaces, $1<p<\infty$. For $p>1, q>1, E=L_{p}$, so that $E^{*}=L_{q}\left(\frac{1}{p}+\frac{1}{q}=1\right)$. Then (see, e.g., $[1$, p. 47$\left.]\right)$ :

$$
\delta_{E^{*}}(\epsilon)=1-\left[1-\left(\frac{\epsilon}{2}\right)^{q}\right]^{\frac{1}{q}} \quad(0<\epsilon \leq 2),
$$

and so we obtain $\delta_{E^{*}}^{-1}(\epsilon)=2\left(1-(1-\epsilon)^{q}\right)^{\frac{1}{q}} \leq 2 q^{\frac{1}{q}} \epsilon^{\frac{1}{q}}$, since $(1-\epsilon)^{q}>1-q \epsilon$, for $q>1$. We verify the following:

(ii) $\delta_{E}^{-1}\left(\beta_{n} M_{0}\right) \leq \alpha_{n} \gamma_{0}$;

(iii) $\delta_{E}^{-1}\left(\eta_{n}\right)=\delta_{E}^{-1}\left(\frac{\alpha_{n-1} \beta_{n}}{\alpha_{n} \beta_{n}}-1\right) \rightarrow 0$, as $n \rightarrow \infty$;

(iv) $\frac{\delta_{E}^{-1}\left(\frac{\alpha_{n-1} \beta_{n}}{\alpha_{n} \beta_{n}}-1\right)}{\alpha_{n} \beta_{n}} \rightarrow 0$, as $n \rightarrow \infty$. 
Now, to verify condition (ii), we have

$$
\begin{aligned}
\frac{\delta_{E}^{-1}\left(\beta_{n} M_{0}\right)}{\alpha_{n}} & =\frac{2\left(1-\left(1-\beta_{n} M_{0}\right)\right)^{\frac{1}{p}}}{\alpha_{n}} \\
& \leq \frac{2 p^{\frac{1}{p}}\left(\beta_{n} M_{0}\right)^{\frac{1}{p}}}{\alpha_{n}} \\
& =\frac{2\left(p M_{0}\right)^{\frac{1}{p}}(n+1)^{-b}}{(n+1)^{-a}}=2\left(p M_{0}\right)^{\frac{1}{p}}(n+1)^{a-b} \rightarrow 0, \quad \text { as } n \rightarrow \infty .
\end{aligned}
$$

Next we show that condition (iii) is satisfied:

$$
\begin{aligned}
\delta_{E}^{-1}\left(\frac{\alpha_{n-1} \beta_{n}}{\alpha_{n} \beta_{n}}-1\right) & =2\left[1-\left(1-\frac{\alpha_{n-1} \beta_{n}}{\alpha_{n} \beta_{n-1}}+1\right)^{p}\right]^{\frac{1}{p}} \\
& =2\left[1-\left(2-\frac{\alpha_{n-1} \beta_{n}}{\alpha_{n} \beta_{n-1}}\right)^{p}\right]^{\frac{1}{p}}=2\left[1-\left(2-\frac{n^{-a}(n+1)^{-b}}{(n+1)^{-a} n^{-b}}\right)^{p}\right]^{\frac{1}{p}} \\
& =2\left[1-\left(2-\left(\frac{n+1}{n}\right)^{a}\left(\frac{n}{n+1}\right)^{b}\right)^{p}\right]^{\frac{1}{p}} \\
& \leq 2\left[1-\left(2-\left(1+\frac{1}{n}\right)^{a}\right)^{p}\right]^{\frac{1}{p}} \leq 2\left[1-\left(1-\frac{a}{n}\right)^{p}\right]^{\frac{1}{p}} \\
& \leq 2 p^{\frac{1}{p}}\left(\frac{a}{n}\right)^{\frac{1}{p}} \rightarrow 0, \quad \text { as } n \rightarrow \infty .
\end{aligned}
$$

Finally, we verify (iv). Using (iii), we have

$$
\begin{aligned}
\frac{\delta_{E}^{-1}\left(\frac{\alpha_{n-1} \beta_{n}}{\alpha_{n} \beta_{n}}-1\right)}{\alpha_{n} \beta_{n}} & \leq \frac{2 p^{\frac{1}{p}}\left(\frac{a}{n}\right)^{\frac{1}{p}}}{(n+1)^{-(a+b)}} \\
& =2 p^{\frac{1}{p}}\left(\frac{a}{n}\right)^{\frac{1}{p}}(n+1)^{(a+b)} \\
& \leq 2^{a+b+1}(p a)^{\frac{1}{p}} n^{a+b-\frac{1}{p}} \rightarrow 0, \quad \text { as } n \rightarrow \infty .
\end{aligned}
$$

\section{Numerical illustrations}

In this section, we present numerical examples to compare the convergence of the sequence generated by our algorithm (3.9) with those produced by algorithms (1.8) and (1.9).

Example 1 In Theorems 1.3, 1.4 and 3.4, set $E=\mathbb{R}^{2}$ then $E^{*}=\mathbb{R}^{2}$. Let

$$
F u=\left(\begin{array}{cc}
3 & -1 \\
1 & 5
\end{array}\right)\left(\begin{array}{l}
u_{1} \\
u_{2}
\end{array}\right), \quad K v=\left(\begin{array}{cc}
7 & 2 \\
-2 & 5
\end{array}\right)\left(\begin{array}{l}
v_{1} \\
v_{2}
\end{array}\right)
$$

Then, it is easy to see that $F$ and $K$ are continuous and strongly monotone. Thus, $K$ and $F$ are maximal monotone and vector $u^{*}=(0,0)^{T}$ is the only solution of the equation $u+$ $K F u=0$. In Theorem 1.3, we take $\alpha_{n}=\frac{1}{n}$, in Theorem 1.4, we take $\lambda_{n}=\theta_{n}=\frac{1}{(n+1)^{\frac{1}{2}}}$, and in Theorem 3.4, we take $\alpha_{n}=\frac{1}{(n+1)^{\frac{1}{2}}}, \beta_{n}=\frac{1}{(n+1)^{\frac{1}{4}}}, n=1,2, \ldots$, as our parameters. Clearly, 
Table 1 Values choosing $u^{1}=(1,0)^{T}, v^{1}=(2,1)^{T}$

\begin{tabular}{|c|c|c|c|c|c|c|}
\hline \multirow[b]{2}{*}{$n$} & \multicolumn{2}{|c|}{ Algorithm (1.8) } & \multicolumn{2}{|c|}{ Algorithm (1.9) } & \multicolumn{2}{|l|}{ Algorithm (3.9) } \\
\hline & $\left\|u_{n+1}\right\|$ & $\left\|v_{n+1}\right\|$ & $\left\|u_{n+1}\right\|$ & $\left\|v_{n+1}\right\|$ & $\left\|u_{n+1}\right\|$ & $\left\|v_{n+1}\right\|$ \\
\hline 1 & 1 & 2 & 1 & 2 & 1 & 2 \\
\hline 2 & 5.0 & 9.0 & 3.2426 & 6.0711 & 4.138 & 6.665 \\
\hline 3 & 5.5 & 24.0 & 5.7301 & 21.5393 & 7.464 & 24.944 \\
\hline 10 & 0.0331 & 0.1479 & 153.338 & 562.409 & 231.37 & 998.396 \\
\hline 20 & 0.0002 & 0.0007 & 4.1488 & 17.0121 & 37.730 & 73.156 \\
\hline 41 & $4.780 \times e^{-6}$ & $6.6 \times e^{-6}$ & 0.0862 & 0.0571 & $3.323 \times \mathrm{e}^{-8}$ & $1.064 \times e^{-7}$ \\
\hline 50 & $2.377 \times e^{-6}$ & $2.387 \times e^{-6}$ & 0.0784 & 0.0519 & & sful \\
\hline 100 & $1.703 \times e^{-7}$ & $2.438 \times e^{-8}$ & 0.0561 & 0.0371 & & sful \\
\hline 116 & $9.703 \times e^{-8}$ & $2.043 \times e^{-8}$ & 0.0519 & 0.0343 & \multirow{2}{*}{\multicolumn{2}{|c|}{$\begin{array}{l}\text { successtul } \\
\text { successful }\end{array}$}} \\
\hline 200 & \multicolumn{2}{|c|}{ successful } & 0.03993 & 0.0263 & & \\
\hline 500 & \multicolumn{2}{|c|}{ successful } & 0.02539 & 0.0167 & \multicolumn{2}{|c|}{ successful } \\
\hline 1000 & \multicolumn{2}{|c|}{ successful } & 0.0179 & 0.0118 & \multicolumn{2}{|c|}{ successful } \\
\hline
\end{tabular}

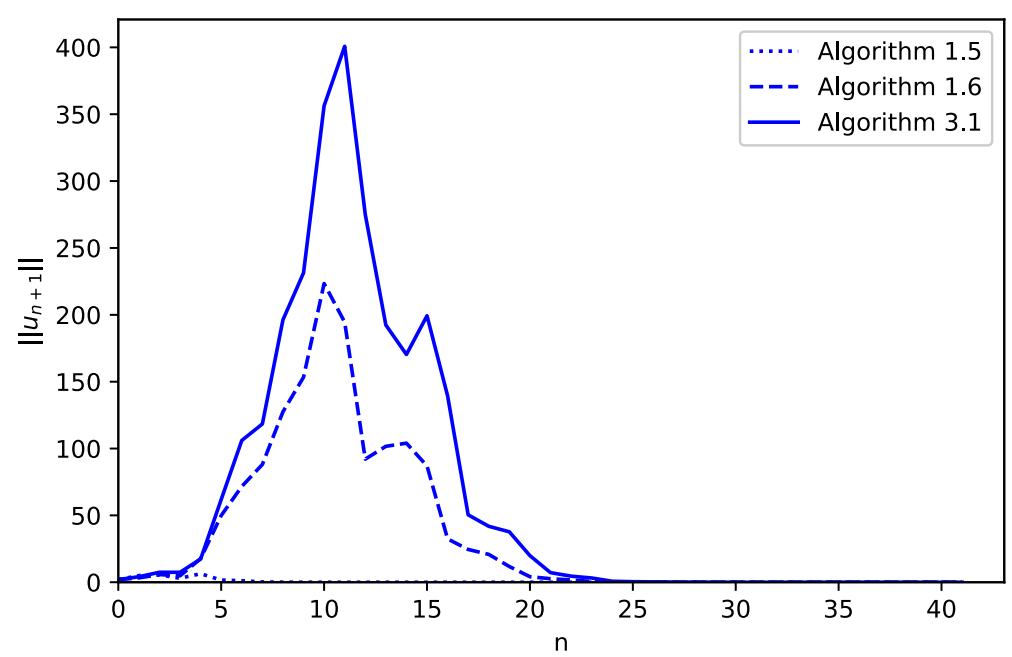

Figure 1 Graph of the first 41 iterates of algorithms (1.8), (1.9) and $(3.9)$, choosing $u^{1}=(1,0)^{T}, v^{1}=(2,1)^{T}$

these parameters satisfy the hypothesis of Theorems 1.3, 1.4, and 3.4. Setting a tolerance of $10^{-8}$ and maximum number of iterations $n=1000$, we obtain the iterates (see Table 1 and Fig. 1).

In Figs. 1, 2, 3 and 4, the $y$-axis represents the values of $\left\|u^{n+1}-\mathbf{0}\right\|$ while the $x$-axis represents the number of iterations $n$.

Example 2 In Theorems 1.3, 1.4, and 3.4, set $E=\mathbb{R}^{2}$ then $E^{*}=\mathbb{R}^{2}$. Let

$$
F u=\left(3 u_{1}-u_{2}+\sin u_{1}, u_{1}+7 u_{2}+\sin u_{2}\right), \quad K v=\left(5 v_{1}-5 v_{2}, 3 v_{1}+6 v_{2}\right) .
$$

Then, it is easy to see that $F$ and $K$ are continuous and strongly monotone. Thus, $K$ and $F$ are maximal monotone and vector $u^{*}=(0,0)^{T}$ is the only solution of the equation $u+$ $K F u=0$. In Theorem 1.3, we take $\alpha_{n}=\frac{1}{n}$, in Theorem 1.4, we take $\lambda_{n}=\theta_{n}=\frac{1}{(n+1)^{\frac{1}{2}}}$, and in Theorem 3.4, we take $\alpha_{n}=\frac{1}{(n+1)^{\frac{1}{2}}}, \beta_{n}=\frac{1}{(n+1)^{\frac{1}{4}}}, n=1,2, \ldots$, as our parameters. Clearly, these parameters satisfy the hypothesis of Theorems 1.3, 1.4, and 3.4. Setting a tolerance 
Table 2 Values choosing $u^{1}=\left(0,-\frac{1}{4}\right)^{T}, v^{1}=\left(-\frac{1}{2}, 1\right)^{T}$

\begin{tabular}{|c|c|c|c|c|c|c|}
\hline \multirow[b]{2}{*}{$n$} & \multicolumn{2}{|c|}{ Algorithm (1.8) } & \multicolumn{2}{|c|}{ Algorithm (1.9) } & \multicolumn{2}{|l|}{ Algorithm (3.9) } \\
\hline & $\left\|u_{n+1}\right\|$ & $\left\|v_{n+1}\right\|$ & $\left\|u_{n+1}\right\|$ & $\left\|v_{n+1}\right\|$ & $\left\|u_{n+1}\right\|$ & $\left\|v_{n+1}\right\|$ \\
\hline 1 & 0.25 & 1 & 0.25 & 1 & 0.25 & 1 \\
\hline 2 & 2.747 & 7.0 & 1.869 & 4.8033 & 2.018 & 5.101 \\
\hline 3 & 8.31 & 18.25 & 7.7957 & 16.312 & 8.738 & 19.059 \\
\hline 10 & 0.683 & 4.419 & 914.49 & 2771.05 & 1040.71 & 3389.62 \\
\hline 20 & 0.0224 & 0.0409 & 1362.57 & 8426.81 & 3612.04 & $13,675.89$ \\
\hline 50 & $6.307 \times e^{-5}$ & 0.0004 & 0.0929 & 0.2106 & 0.0718 & 0.2818 \\
\hline 80 & $1.678 \times e^{-5}$ & $2.23 \times e^{-5}$ & 0.0015 & 0.0161 & $9.382 \times e^{-8}$ & $2.875 \times e^{-8}$ \\
\hline 383 & $1.106 \times e^{-8}$ & $5.064 \times e^{-9}$ & 0.0007 & 0.0075 & \multicolumn{2}{|c|}{ successful } \\
\hline 500 & \multicolumn{2}{|c|}{ successful } & 0.0006 & 0.0065 & \multicolumn{2}{|c|}{ successful } \\
\hline 1000 & \multicolumn{2}{|c|}{ successful } & 0.0004 & 0.0046 & & ssful \\
\hline
\end{tabular}

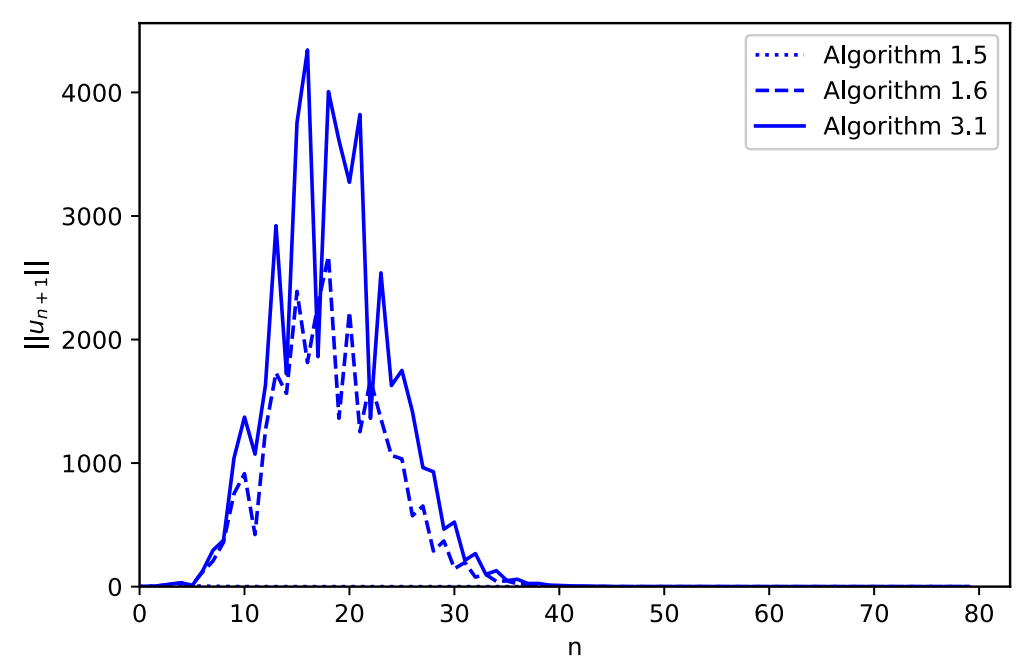

Figure 2 Graph of the first 80 iterates of algorithms (1.8), (1.9), and (3.9), choosing $u^{1}=\left(0,-\frac{1}{4}\right)^{\top}, v^{1}=\left(-\frac{1}{2}, 1\right)^{\top}$

of $10^{-8}$ and maximum number of iterations $n=1000$, we obtain the iterates (see Table 2 and Fig. 2).

In Theorems 1.4 and 3.4, set $E=L_{p}([0,1]), E^{*}=L_{q}([0,1]), 1<p<q<\infty$, and $\frac{1}{p}+\frac{1}{q}=1$.

$$
\begin{aligned}
F: L_{p}([0,1]) & \rightarrow L_{q}([0,1]), \\
u & \longmapsto(F u)(t)=J u(t) .
\end{aligned}
$$

It is well known that the normalized duality map $J$ is monotone and uniformly continuous on bounded subsets of $L_{p}$ and thus, maximal monotone. Let

$$
\begin{aligned}
K: L_{q}([0,1]) & \rightarrow L_{p}([0,1]), \\
v & \longmapsto(K v)(t)=t v(t) .
\end{aligned}
$$


Since $L_{q}([0,1]) \subset L_{p}([0,1]), K$ is well-defined. Observe that by definition $K$ is linear. Next we show that $K$ is monotone. Let $v, w \in L_{q}([0,1])$, then

$$
\begin{aligned}
\langle(K v)(t)-(K w)(t), v(t)-w(t)\rangle & =\int_{0}^{1}(t v(t)-t w(t))(v(t)-w(t)) d t \\
& =\int_{0}^{1} t(v(t)-w(t))^{2} d t \geq 0 .
\end{aligned}
$$

Hence, $K$ is monotone. Furthermore, since $K$ is continuous, $K$ is maximal monotone and the function $u^{*}(t)=(0,0)^{T}$ is the only solution of the equation $u+K F u=0$. For the numerical experiments, in Examples 3 and 4 below, in Theorem 1.4, we take $\lambda_{n}=\theta_{n}=\frac{1}{(n+1)^{\frac{1}{2}}}$, and in Theorem 3.4, we take $\alpha_{n}=\frac{1}{(n+1)^{\frac{1}{2}}}, \beta_{n}=\frac{1}{(n+1)^{\frac{1}{4}}}, n=1,2, \ldots$, as our parameters. Clearly, these parameters satisfy the hypothesis of Theorems 1.4 and 3.4. We set a tolerance of $10^{-2}$ and maximum number of iterations $n=10$.

\begin{tabular}{|c|c|c|c|c|}
\hline \multirow[b]{2}{*}{$n$} & \multicolumn{2}{|c|}{$\begin{array}{l}\text { Algorithm (1.9) } \\
\text { Time }=38.168 \mathrm{~s}\end{array}$} & \multicolumn{2}{|c|}{$\begin{array}{l}\text { Algorithm (3.9) } \\
\text { Time }=33.342 \mathrm{~s}\end{array}$} \\
\hline & $\left\|u_{n+1}\right\|$ & $\left\|v_{n+1}\right\|$ & $\left\|u_{n+1}\right\|$ & $\left\|v_{n+1}\right\|$ \\
\hline 1 & 0.2644 & 0.4005 & 0.2273 & 0.1152 \\
\hline 2 & 0.2411 & 0.4674 & 0.0535 & 0.3433 \\
\hline 3 & 0.3784 & 0.3742 & 0.3407 & 0.2467 \\
\hline 4 & 0.4156 & 0.4267 & 0.4038 & 0.3151 \\
\hline 5 & 0.4518 & 0.3939 & 0.5258 & 0.3004 \\
\hline 6 & 0.5447 & 0.4664 & 0.5794 & 0.3319 \\
\hline 7 & 0.5232 & 0.3954 & 0.6505 & 0.3273 \\
\hline 8 & 0.6153 & 0.4914 & 0.6800 & 0.3405 \\
\hline 9 & 0.5780 & 0.3874 & 0.7354 & 0.3504 \\
\hline 10 & 0.5918 & 0.3074 & 0.8007 & 0.2724 \\
\hline
\end{tabular}

Table 3 Values choosing $u^{1}(t)=t^{2}+1$ and $v^{1}(t)=\cos t \exp (-t)$

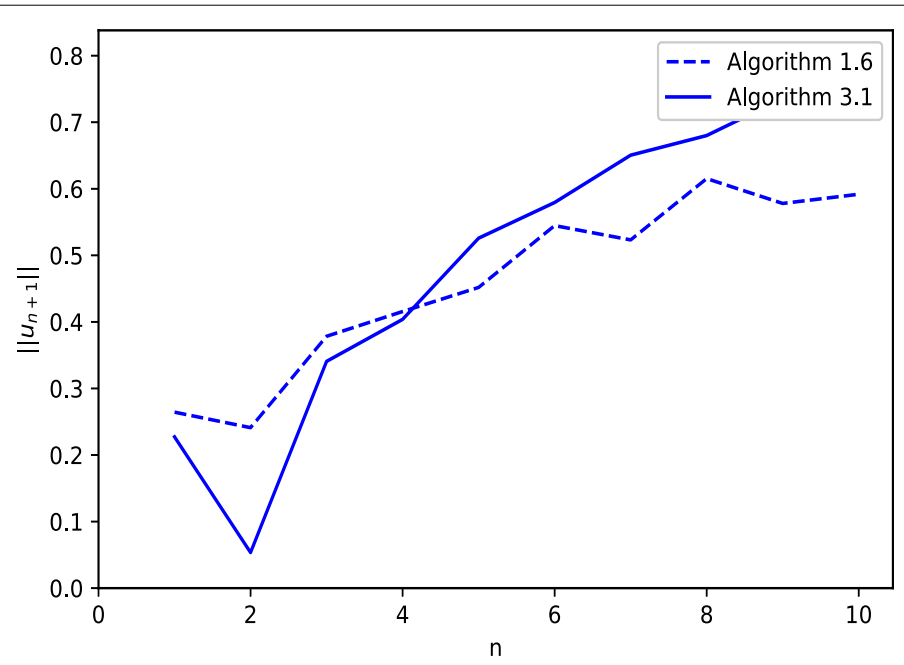

Figure 3 Graph of the first 10 iterates of algorithms (1.9) and (3.9), choosing $u^{1}(t)=t^{2}+1$ and $v^{1}(t)=\cos t \exp (-t)$ 
Example 3 Taking $p=1.5$, we have $E=L_{1.5}([0,1]), E^{*}=L_{3}([0,1])$ (see Table 3 and Fig. 3)

$$
\begin{aligned}
F: L_{1.5}([0,1]) & \rightarrow L_{3}([0,1]), \\
u & \longmapsto(F u)(t)=J u(t), \\
K: L_{3}([0,1]) & \rightarrow L_{1.5}([0,1]), \\
v & \longmapsto(K v)(t)=t v(t) .
\end{aligned}
$$

\begin{tabular}{|c|c|c|c|c|}
\hline \multirow[b]{2}{*}{$n$} & \multicolumn{2}{|c|}{$\begin{array}{l}\text { Algorithm (1.9) } \\
\text { Time }=17.700 \mathrm{~s}\end{array}$} & \multicolumn{2}{|c|}{$\begin{array}{l}\text { Algorithm (3.9) } \\
\text { Time }=14.223 \mathrm{~s}\end{array}$} \\
\hline & $\left\|u_{n+1}\right\|$ & $\left\|v_{n+1}\right\|$ & $\left\|u_{n+1}\right\|$ & $\left\|v_{n+1}\right\|$ \\
\hline 1 & 1.2997 & 0.8496 & 0.8985 & 0.7560 \\
\hline 2 & 0.6109 & 1.8814 & 0.4550 & 1.5637 \\
\hline 3 & 1.4480 & 0.4603 & 0.9682 & 0.2581 \\
\hline 4 & 0.4061 & 1.3936 & 0.4318 & 1.0630 \\
\hline 5 & 1.1624 & 0.7364 & 0.8631 & 0.7177 \\
\hline 6 & 0.7735 & 1.1298 & 0.7839 & 0.9367 \\
\hline 7 & 1.0413 & 0.9851 & 0.9025 & 0.9110 \\
\hline 8 & 0.9748 & 1.0935 & 0.9261 & 0.9713 \\
\hline 9 & 1.0519 & 1.0873 & 0.9713 & 0.9977 \\
\hline 10 & 1.0611 & 1.1242 & 1.0002 & 1.0301 \\
\hline
\end{tabular}

Example 4 Taking $p=\frac{5}{3}$, we have $E=L_{\frac{5}{3}}([0,1]), E^{*}=L_{2.5}([0,1])($ see Table 4 and Fig. 4$)$

$$
\begin{aligned}
F: L_{\frac{5}{3}}([0,1]) & \rightarrow L_{2.5}([0,1]), \\
u & \longmapsto(F u)(t)=J u(t), \\
K: L_{2.5}([0,1]) & \rightarrow L_{\frac{5}{3}}([0,1]), \\
v & \longmapsto(K v)(t)=t v(t) .
\end{aligned}
$$

Table 4 Values choosing $u^{1}(t)=\frac{1}{1+x \sin x}$ and $v^{1}(t)=\exp (t)$

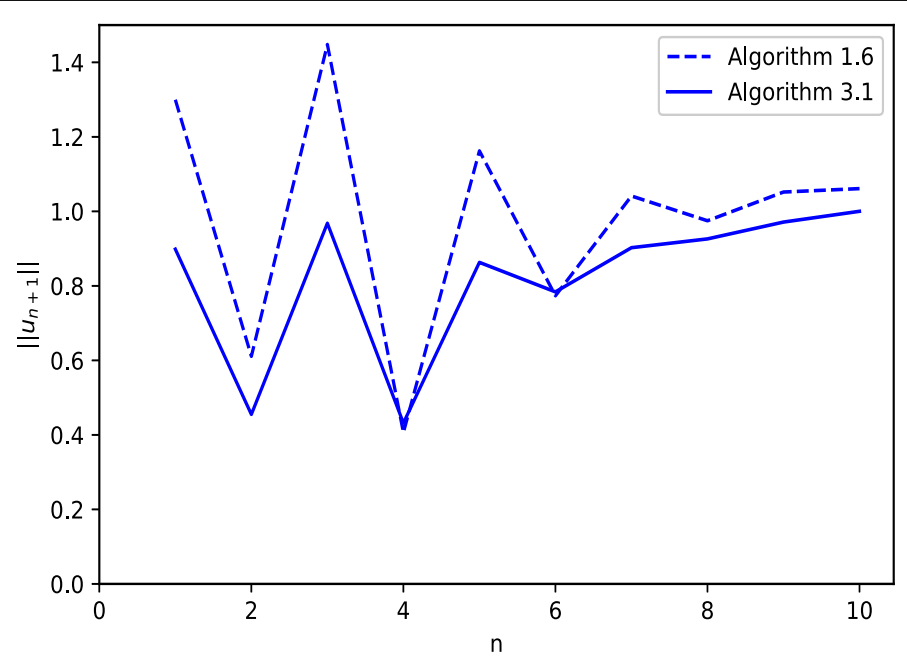

Figure 4 Graph of the first 10 iterates of algorithms (1.9) and (3.9), choosing $u^{1}(t)=\frac{1}{1+x \sin x}$ and $v^{1}(t)=\exp (t)$ 
Remark 8 From the numerical experiments above, we see that algorithm (3.9) is more robust and efficient than algorithms (1.8) and (1.9), and converges faster in terms of number of iterations and CPU time in all the problems tested.

\section{Conclusion}

In this paper, an iterative algorithm that extends the results of Chidume and Shehu [24], and complements the results of Uba et al. [44] is constructed. Strong convergence of the sequence generated by the algorithm is proved in a uniformly convex and uniformly smooth real Banach space. The theorem proved is a significant improvement of the results of Chidume and Shehu [24] which was proved in real Hilbert spaces under the assumption that $F$ and $K$ are continuous and bounded. These restrictions on $K$ and $F$ have been dispensed with even in the more general setting considered here. Finally, numerical experiments are presented to demonstrate the convergence of the sequence of the proposed algorithm.

\section{Acknowledgements}

The authors appreciate the support of their institute and AfDB for the Research Grant that enabled this work to be carried out. The authors wish to thank the referees for their esteemed comments and suggestions.

\section{Funding}

This work is supported from AfDB Research Grant Funds to AUST.

Availability of data and materials

Not applicable.

\section{Competing interests}

The authors declare that they have no conflict of interest.

\section{Authors' contributions}

CEC formulated the problem and suggested the method of proof of the Theorem to AA and LCO. The computations using the method suggested by CEC were carried out by AA and LCO. The analysis of the computations to arrive at the proof of the theorem was done jointly by CEC, AA and LCO. All authors read and approved the final manuscript.

\section{Publisher's Note}

Springer Nature remains neutral with regard to jurisdictional claims in published maps and institutional affiliations.

Received: 25 September 2019 Accepted: 19 January 2020 Published online: 17 February 2020

\section{References}

1. Alber, Y., Ryazantseva, I.: Nonlinear III Posed Problems of Monotone Type. Springer, London (2006)

2. Berinde, V.: Iterative Approximation of Fixed Points. Lecture Notes in Mathematics. Springer, London (2007)

3. Brezis, H., Browder, F.E.: Some new results about Hammerstein equations. Bull. Am. Math. Soc. 80, 567-572 (1974)

4. Brezis, H., Browder, F.E.: Existence theorems for nonlinear integral equations of Hammerstein type. Bull. Am. Math. Soc. 81, 73-78 (1975)

5. Brezis, H., Browder, F.E.: Nonlinear integral equations and system of Hammerstein type. Adv. Math. 18, 115-147 (1975)

6. Browder, F.E., Gupta, P.: Monotone operators and nonlinear integral equations of Hammerstein type. Bull. Am. Math. Soc. 75, 1347-1353 (1969)

7. Chepanovich, R.S.: Nonlinear Hammerstein equations and fixed points. Publ. Inst. Math. (Belgr.) 35, 119-123 (1984)

8. Chidume, C.E.: Iterative solution of the equation $f \in x+T x$ for a monotone operator in $L_{p}$ spaces. J. Math. Anal. Appl. $116(2), 531-537(1986)$

9. Chidume, C.E.: Iterative solution of nonlinear equations of the monotone type in Banach spaces. Bull. Aust. Math. Soc. 42(1), 21-31 (1990)

10. Chidume, C.E.: Geometric Properties of Banach Spaces and Nonlinear Iterations. Lectures Notes in Mathematics, vol. 1965. Springer, London (2009)

11. Chidume, C.E., Adamu, A., Chinwendu, L.O.: A Krasnoselskii-type algorithm for approximating solutions of variational inequality problems and convex feasibility problems. J. Nonlinear Var. Anal. 2(2), 203-218 (2018)

12. Chidume, C.E., Adamu, A., Chinwendu, L.O.: Approximation of solutions of Hammerstein equations with monotone mappings in real Banach spaces. Carpath. J. Math. 35(3), 305-316 (2019)

13. Chidume, C.E., Bello, A.U.: An iterative algorithm for approximating solutions of Hammerstein equations with monotone maps in Banach spaces. Appl. Math. Comput. 313, 408-417 (2017)

14. Chidume, C.E., Chidume, C.O.: Convergence theorem for zeros of generalized Lipschitz generalized Phi-accretive operators. Proc. Am. Math. Soc. 134(1), 243-251 (2006) 
15. Chidume, C.E., Djitte, N.: Iterative approximation of solutions of nonlinear equations of Hammerstein-type. Nonlinear Anal. 70, 4086-4092 (2009)

16. Chidume, C.E., Djitte, N.: Approximation of solutions of Hammerstein equations with bounded strongly accretive nonlinear operator. Nonlinear Anal. 70, 4071-4078 (2009)

17. Chidume, C.E., Djitte, N.: An iterative method for solving nonlinear integral equations of Hammerstein type. Appl. Math. Comput. 219, 5613-5621 (2013)

18. Chidume, C.E., Idu, K.O.: Approximation of zeros of bounded maximal monotone maps, solutions of Hammerstein integral equations and convex minimization problems. Fixed Point Theory Appl. (2016). https://doi.org/10.1186/s13663-016-0582-8

19. Chidume, C.E., Ikechukwu, S.I., Adamu, A.: Inertial algorithm for approximating a common fixed point for a countable family of relatively nonexpansive maps. Fixed Point Theory Appl. (2018). https://doi.org/10.1186/s13663-018-0634-3

20. Chidume, C.E., Nnakwe, M.O., Adamu, A.: A strong convergence theorem for generalized- $\boldsymbol{\Phi}$-strongly monotone maps, with applications. Fixed Point Theory Appl. (2019). https://doi.org/10.1186/s13663-019-0660-9

21. Chidume, C.E., Ofoedu, E.U.: Solution of nonlinear integral equations of Hammerstein type. Nonlinear Anal. 74, 4293-4299 (2011)

22. Chidume, C.E., Shehu, Y.: Approximation of solutions of generalized equations of Hammerstein type. Comput. Math. Appl. 63, 966-974 (2012)

23. Chidume, C.E., Shehu, Y.: Iterative approximation of solutions of equations of Hammerstein type in certain Banach spaces. Appl. Math. Comput. 219, 5657-5667 (2013)

24. Chidume, C.E., Shehu, Y.: Approximation of solutions of equations Hammerstein type in Hilbert spaces. Fixed Point Theory 16(1), 91-102 (2015)

25. Chidume, C.E., Zegeye, H.: Iterative approximation of solutions of nonlinear equation of Hammerstein-type. Abstr. Appl. Anal. 6, 353-367 (2003)

26. Chidume, C.E., Zegeye, H.: Approximation os solutions of nonlinear equations of monotone and Hammerstein-type. Appl. Anal. 82(8), 747-758 (2003)

27. Chidume, C.E., Zegeye, H.: Approximation of solutions nonlinear equations of Hammerstein type in Hilbert space. Proc. Am. Math. Soc. 133, 851-858 (2005)

28. De Figueiredo, D.G., Gupta, C.P.: On the variational methods for the existence of solutions to nonlinear equations of Hammerstein type. Bull. Am. Math. Soc. 40, 470-476 (1973)

29. Djitte, N., Sene, M.: An iterative algorithm for approximating solutions of Hammerstein integral equations. Numer. Funct. Anal. Optim. 34(12, 1299-1316 (2013)

30. Dolezale, V:: Monotone Operators and Its Applications in Automation and Network Theory, Studies in Automation and Control. Elesevier, New York (1979)

31. Goebel, K., Reich, S.: Uniform Convexity, Hyperbolic Geometry, and Nonexpansive Mappings. Monographs and Textbooks in Pure and Applied Mathematics, vol. 83. Marcel Dekker, New York (1984)

32. Kamimura, S., Takahashi, W.: Strong convergence of a proximal-type algorithm in a Banach space. SIAM J. Optim. 13, 938-945 (2002)

33. Lindenstrauss, J., Tzafriri, L.: Classical Banach Spaces II: Function Spaces. Ergebnisse Math. Grenzgebiete Bd., vol. 97. Springer, Berlin (1979)

34. Minjibir, M.S., Mohammed, I.: Iterative algorithms for solutions of Hammerstein integral inclusions. Appl. Math. Comput. 320, 389-399 (2018)

35. Minty, G.J: Monotone (nonlinear) operators in Hilbert space. Duke Math. J. 29(4), 341-346 (1962)

36. Narendra, K., Gallman, P.: An iterative method for the identification of nonlinear systems using a Hammerstein model. In: IEEE Transactions on Automatic Control, vol. 11, pp. 546-550 (1966). https://doi.org/10.1109/TAC.1966.1098387

37. Nilsrakoo, W., Saejung, S.: Strong convergence theorems by Halpern-Mann iterations for relatively nonexpansive maps in Banach spaces. Appl. Math. Comput. 217, 6577-6586 (2011)

38. Ofoedu, E.U., Malonza, D.M.: Hybrid approximation of solutions of nonlinear operator equations and application to equation of Hammerstein type. Appl. Math. Comput. 217, 6019-6030 (2011)

39. Ofoedu, E.U., Onyi, C.E.: New implicit and explicit approximation methods for solutions of integral equations of Hammerstein type. Appl. Math. Comput. 246, 628-637 (2014)

40. Pascali, D., Sburlan, S.: Nonlinear Mappings of Monotone Type. Editura Academiei, Bucharest (1978)

41. Reich, S.: Constructive techniques for accretive and monotone operators. In: Applied Nonlinear Analysis, pp. 335-345 Academic Press, New York (1979)

42. Rockafellar, R.T.: Local boundedness of nonlinear monotone operators. Mich. Math. J. 16, 397-407 (1969)

43. Shehu, Y.: Strong convergence theorem for integral equations of Hammerstein type in Hilbert spaces. Appl. Math. Comput. 231, 140-147 (2014)

44. Uba, M.O., Uzochukwu, M.I., Onyido, M.A.: Algorithm for approximating solutions of Hammerstein integral equations with maximal monotone operators. Indian J. Pure Appl. Math. 48(3), 391-410 (2017)

45. Xu, H.K.: Iterative algorithms for nonlinear operators. J. Lond. Math. Soc. (2) 66, 240-256 (2002)

46. Zarantonello, E.H.: Solving functional equations by contractive averaging. U.S. Army Mathematics Research Center. Technical Report 160

47. Zeidler, E.: Nonlinear Functional Analysis and Its Applications II/B: Nonlinear Monotone Operators. Springer, New York (1986) 